\title{
UNDERSTANDING THE CORPUS OF MOBILE PAYMENT SERVICES RESEARCH: AN ANALYSIS OF THE LITERATURE USING CO-CITATION ANALYSIS AND SOCIAL NETWORK ANALYSIS
}

Surabhi Verma, http://orcid.org/0000-0001-7641-0637

Southern Denmark University, Odense, Denmark

Sushil S. Chaurasia, http://orcid.org/0000-0003-0747-8907

University of New Brunswick, Fredericton, Canada

Vibhav Singh, http://orcid.org/0000-0001-8810-2631

Narsee Monjee Institute of Management Studies University - NMIMS, Navi Mumbai, India

\begin{abstract}
Mobile Payment Services have advanced in the last two decades, gaining the attention of experts and researchers from around the world. A number of reviews and literature analysis studies have been carried out, aimed at analysing the numerous dimensions of mobile payment services; however, no researcher has attempted a co-citation analysis to scrutinise and comprehend the core knowledge structures that are integral parts of mobile payment services studies. Therefore, in order to fill this research gap, this research article aims to interpret the corpus of mobile payment services research, which was published during the period of 1997 to June 2017. Bibliometric and Social Network Analysis (SNA) methods were employed to formulate the core intellectual structure of research targeting mobile payment services. The Web of Knowledge (WoK) database was the key source from where 406 articles and 3,424 citations were obtained. These documents were analysed using co-citation analysis. UCINET was used to enlist the keynote research papers in the realm of mobile payment services as per factor analysis, citation and cocitation analysis, multidimensional scaling and centrality measurement. Seven core clusters of mobile payment services research emerged as a critical finding of this study; these clusters include (1) Adoption and usage; (2) Trust, risk and security; (3) Application; (4) Scheme; (5) Protocol; (6) Architecture; (7) Mobile payment corporation. The findings of this research study provide crucial guidelines for practitioners and researchers involved in this field.
\end{abstract}

Keywords: Mobile Payment Services; Citation; Co-citation; Social Network Analysis.

Manuscript first received: 2018/11/16. Manuscript accepted: 2019/10/04

Surabhi Verma, Department of Marketing and Management Centre for integrative innovation management, Southern Denmark University, Odense, Denmark. Email: verma9016@gmail.com

Sushil S. Chaurasia, The J Herbert Smith Centre for Technology Management \& Entrepreneurship University of New Brunswick Fredericton, E3B 5A3, Canada. Email: chaurasia.sushil@gmail.com

Vibhav Singh, Narsee Monjee Institute of Management Studies University - NMIMS, Navi Mumbai, India.

Email: drvibhav1108@gmail.com 


\section{INTRODUCTION}

With the development of mobile phone, fixed-line telephony billing systems had to be upgraded to accommodate mobile telephony billing systems for products or services buying and selling (Keramati et al., 2012). Billing system-based payments can suffer from numerous challenges, including inflated payment transaction charges, the necessity to provision services to billing systems and unfair revenue sharing (Koenig-Lewis et al., 2015). To overcome these problems, mobile payment services emerged as a new payment instrument for fund transfer (Isaac and Zeadally, 2014). Mobile payment services serve as the primary delivery platform for funds transfer or payments for services, goods or bills with mobile devices like smartphone or personal digital devices (PDA) and mobile internet (MolinaCastillo et al., 2016). Mobile payment services permit users to execute financial transactions over their mobile phones in a manner that is secure, simple and serviceable (Isaac and Zeadally, 2014). It covers different types of payment like fares for taxis, buses or train, miscellaneous digital products such as apps, games, music etc., and tickets for movies and flight (Molina-Castillo et al., 2016). Instruments such as credit card, e-wallet or mobile wallet can be used to close financial transactions over mobile phones.

In the past two decades, mobile payment services have become attractive topics of research for scholars and practitioners alike. However, even after twenty years of the acceptance of mobile payment services and their globally pervasive usage, the question remains as to whether mobile payment services have attained maturity and if they have, what aspects need to be further researched. The Web of Knowledge (WoK) database stands testimony to the popularity of mobile payment services as a research topic. A number of reviews and literature analysis studies have been published to ascertain the manifold dimensions of mobile payment services. However, most of the previous studies did not target publications about high-value mobile payment services or the core intellectual structures of this research topic. This research gap can be filled using bibliometric and social network analysis (a mathematical and statistical approach), pinpointing the consistent study areas in the field (Sugimoto et al., 2008). Citation and co-citation analyses are popular bibliometric tools (Borgman 1989). Citation analysis is a popular method to evaluate the popularity and influence exerted by individual authors, articles or publications (Shiau, 2015). On the other hand, co-citation analysis yields an objective and quantifiable way to emphasize the intellectual core within any given research field (Pilkington and Meredith 2009). Also, this research article yields in-depth insights into the structure of the research, managed through UCINET that used factor analysis, multidimensional scaling and centrality measurement to the literature database. Rest of the present research article has been organised as follows. The literature review section reviews mobile payment services literature. The methodology section expounds the various methods employed in this study in detail, and the analytical procedure employed, apart from the dataset elements of mobile payment services publications. Next, the critical findings of the analysis have been presented, followed by a discussion of the main outcomes. The conclusions summarise the main findings and their crucial implications.

\section{LITERATURE REVIEW}

\section{Mobile Payment Services}

Mobile payment services, which originated in late 90's, eliminates or reduces the need of cash (Pham and Ho, 2015), offers convenience and speed (Teo et al., 2015) and provides secure transfer of 
information (Schierz et al., 2010). Mobile payment services refers to business or individual activities that utilizes digital devices with mobile internet, in order to perform any economic transactions (Liébana-Cabanillas et al., 2014 (b)). Unlike the spatial and temporal constraints in both offline and online payments (Zhou, 2013 (b)), mobile payment services enables users to complete their payments in a faster, safer and more convenience transactions at anywhere and anytime (Liébana-Cabanillaset al., 2014; Ondrus and Pigneur, 2009; Zhou, 2013 (a)). As the usage of smart phone and mobile internet increases, mobile payment services grew rapidly. Both consumers and business are benefited from this mode of payment (Madan and Yadav, 2016). From consumers' perspective, the benefits of mobile payment services includes convenience (Mallat and Tuunainen, 2008), purchases independent of location and time (Constantiou et al., 2006; Mallat et al., 2009), person to person money transfer, ticketing and loyalty programmes (Mallat et al., 2009). In addition, the benefits of mobile payment services to retailers includes faster services, lower transaction costs and decrease in the number of cash transactions (Mallat and Tuunainen, 2008). All these benefits of mobile payment services makes it the next step of electronic payment services evolution and has tremendous potential to grow (Kim et al., 2010 (a)). Due to these reasons, practitioners and academicians started looking into this new innovation and the number of studies of mobile payment services has increased tremendously.

Mobile payment services knowledge is often narrowly defined as exchange of financial values in return for services and goods (Ooi and Tan, 2016). To better understand the nature and scope of mobile payment services, a number of studies reviewed the literature, proposed a framework, or classified mobile payment services articles. For example, Dahlberg et al. (2008) proposed a framework for mobile payment services market based on generic contingency theory and competitive forces. The framework has four outer factors including changing social/cultural, commercial and technical and legal/ regulatory/ standards environment. The inner facet of the framework describes the five main competitive forces of mobile payment services including traditional payment services, merchant power, new E-payment services and consumer power. Ngai and Gunasekaran (2007) performed an extensive literature review and identified 149 research articles on m-commerce. Their results classified the $\mathrm{m}$-commerce literature into $\mathrm{m}$-commerce theory and research, wireless network infrastructure, mobile middleware, wireless user infrastructure and m-commerce application and cases. Recently, Shaikh and Karjaluoto (2015) systematically reviewed the literature on m-banking adoption studies published between January 2005 and March 2014. The results identified 55 relevant studies and identified that m-banking adoption literature mostly relies on the technology acceptance model and its modification. The most significant drivers of m-banking services adoption intention are compatibility, perceived usefulness and attitude. In last years, mobile payment services has also covered more recent topics, including near field communication (de Kervile et al., 2016), e-wallet (Shin, 2010), business model innovation issues (Ramos-de-Luna et al., 2016).

\section{METHODOLOGY}

The systematic method of reviewing the mobile payment services literature is planned in three phases (Figure 1). Dataset were compiled for mobile payment services publications, in the first phase. In the second phase, citation analysis and co-citation analysis techniques were used to get the intellectual structure of mobile payment services research domain. In the third and last phase, the methodology of social network analysis was employed to interpret the relationship among articles. All the above-mentioned phases have been elaborated upon in the ensuing sections. 


\section{First phase-Data Collection}

Web of Knowledge (WoK) is among the most prolific research databases, offering citations and publications from as many as 10,000 journals held in high esteem by experts (Shiau, 2015). Fernandes et al. (2017) opine that WoK is one of the most widely used databases across the world. Several researches use this database to ascertain related information, objectively and methodically.

Data retrieval from the Web of Knowledge (WoK) database was carried out to assess the level of baseline research conducted so far in the field of mobile payment services. Keywords such as "mobile payment services", "m-payment" and "wireless payment" were employed in the exploration phase of the study to find and stratify representative articles in the database under the title, abstract or publication keyword section. The articles carefully chosen for this research were in the English language and were published in journals. Also, one level of manual scrutiny was executed to ascertain that only publications related to mobile payment services had been chosen. The result of this exercise was a list of carefully curated research articles associated with mobile payment services research.

\section{Second phase-Citation and Co-citation analysis}

In this stage of the study, the co-citation analysis method, as outlined by White and Griffith (1981) was espoused to examine the intellectual structure of mobile payment services literature. As per Small (1973), co-citation "is a frequency count of earlier publications cited together in later publication". Many scholars (such as White and Griffith, 1981; Pilkington and Meredith, 2009; Hsiao and Yang, 2011; Shiau and Dwivedi, 2013) make use of co-citation analysis in their studies to conclude upon the intellectual structure of research. Likenesses between the selected evaluation units can be emphasized using the technique of co-citation analysis, which can be applied with ease to any given analysis unit, be it author, journal or document. While the author co-citation analysis approach is employed to quantify the core knowledge themes of information science (White and Griffith, 1981), journal co-citation studies do the same for economics journals (Mccain, 1991).On the other hand, document co-citation analysis puts forth articles that are products of superior research (Webster \& Watson 2002); such articles are inarguably more reliable, being qualified on the basis of a detailed review (Ramos-Rodríguez and Ruíz-Navarro 2004).

However, despite its multifarious beneficial aspects, co-citation analysis does come with its own limitations. As per Shiau, (2015), author co-citation analysis is biased as it omits all authors but for the first one (). Also, self-citation by authors is not eliminated. In addition, it frequently occurs that a given set of authors continue to work in a given research area and end up with multiple publications in this field (Fernandes et al., 2017).

Dual sets of articles are employed to formulate a co-citation matrix when conducting document co-citation analysis (Khan and Wood, 2015). Between these two, the first set comprises of articles that have been cited, i.e., these are the source or published articles; on the other hand, the second set of articles are those that cite the source articles.

The second phase commenced by compiling the data of cited articles from the first phase and meta-data of citing articles obtained from the Web of Knowledge website. Post this, the frequency of co-citation for each pair of cited articles was calculated and the same was converted into a square symmetrical co-citation matrix, treating the diagonal value as zero to eliminate any self-citations (McCain 1990; Ramos-Rodríguez \& Ruíz-Navarro 2004). Therefore, this research took up document 
PHASE

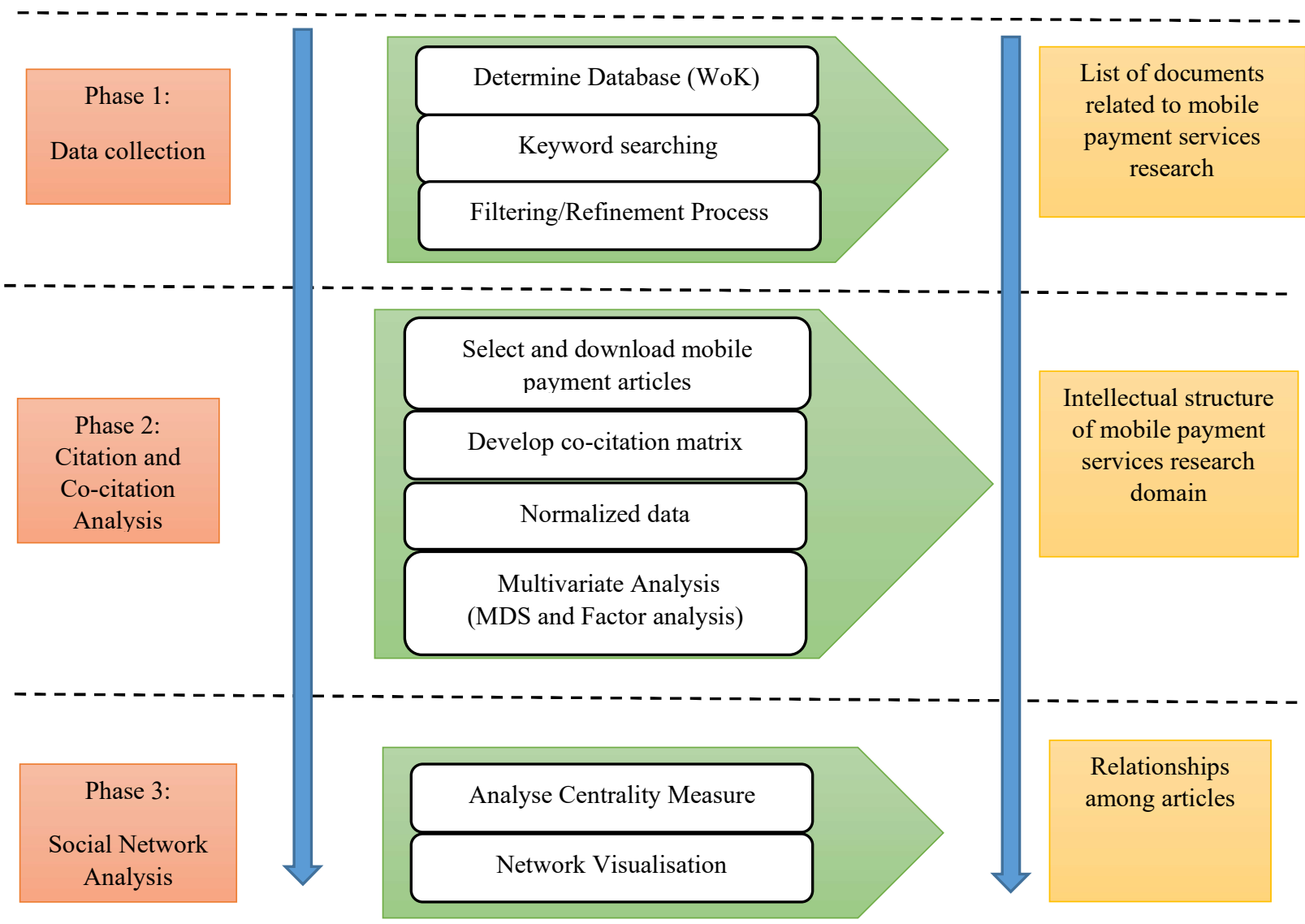

Figure 1: Systematic Process for Reviewing the M-Payment Literature

co-citation analysis given that it is a more precise approach towards examining the intellectual structure of mobile payment services research.

Pearson's correlation coefficient for multivariate analysis was employed in order to normalize the co-citation matrix (Hsiao and Yang, 2011). Here, Pearson's correlation coefficient was given preference because of the computation of degree of similarity, which necessitates the likeness relationship between each and every one of the articles (Fernandes et al., 2017). In addition, this addresses the concern of unlimited divergence between two related documents, which occurs due to differences in the scale (White \& Mccain 1998). Normalized co-citation matrix is employed to implement factor analysis to stratify the basic themes of mobile payment services research (Tabachnick and Fidell, 2008). Factor loadings higher than \pm 0.7 were used to detect the most suitable items (McCain, 1990; Hair et al., 1998). The NetDraw tool was employed to present a visually appealing representation of the outcomes of factor analysis outcomes (Borgatti 2002).

\section{Third phase}

Social network analysis makes use of the centrality measure for ascertaining the most critical and influential documents (or nodes) in the network map. UCINET was used to formulate a co- 
citation network map (Borgatti et al., 2002), which was used to quantify the proportional relationships present between the articles (Wang and Chen, 2014). Degree centrality, betweenness centrality and eigenvector centrality were used as the three measures of social network analysis.

- Degree centrality: This may be called as local centrality as well. It emphasizes the popularity ranking of an article. In the present study, Degree Centrality was used to assess the number of direct links between documents (Khan and Wood, 2015).

- Betweenness centrality: Betweenness Centrality is employed to compute how many times an article is used as the shortest path between two other documents (Hu et al., 2013). Higher this score, greater the influence the article exerts on the network (Hu et al., 2013). Articles with high betweenness centrality value are considered to be the pioneering ones, with the probability of managing network process (Khan and Wood, 2015).

- Eigenvector centrality: This is used to compute the influence of the central document that is strongly interlinked with the other principal articles (Bonacich, 1972). The influence this document has on other critical articles in the research domain is directly proportional to the value of eigenvector centrality.

\section{RESULTS}

\section{Data}

The keywords/strings employed during the study were "mobile payment services", "m-payment", "wireless payment"; these keywords could be observed in the title, abstract or keywords sections of the WoK database. English language articles published in journals during 1997 to 2017 were included in this study. As many as 1,002 bibliographic references were obtained using the above-mentioned keywords.

\section{Descriptive analysis}

The number of mobile payment services articles published in research journals shows a gradual incline (Figure 2). Overall, 1,002 research documents had been published during 1997 to 2017, out these articles only 411 were published in journals between 1997 and 2017. After restricting language to English only the total number of research articles reduced to 406 only. The number of mobile payment services research articles started with one article in 1997, progressing gradually over the next 19 years. Two insignificant drops in the number were observed in the years 2004 and 2007. This reflects the mounting interest in and acceptance and adoption of mobile payment services all over the world.

The five articles containing the greatest number of citations are respectively:

- Zhou, T., Lu, Y., \& Wang, B. (2010). Integrating TTF and UTAUT to explain mobile banking user adoption. Computers in human behavior, 26(4), 760-767.

- Mallat, N. (2007). Exploring consumer adoption of mobile payments-A qualitative study. The Journal of Strategic Information Systems, 16(4), 413-432. 
- Kim, C., Mirusmonov, M., \& Lee, I. (2010). An empirical examination of factors influencing the intention to use mobile payment. Computers in Human Behavior, 26(3), 310-322.

- Schierz, P. G., Schilke, O., \& Wirtz, B. W. (2010). Understanding consumer acceptance of mobile payment services: An empirical analysis. Electronic commerce research and applications, 9(3), 209-216.

- Chen, L. D. (2008). A model of consumer acceptance of mobile payment. International Journal of Mobile Communications, 6(1), 32-52.

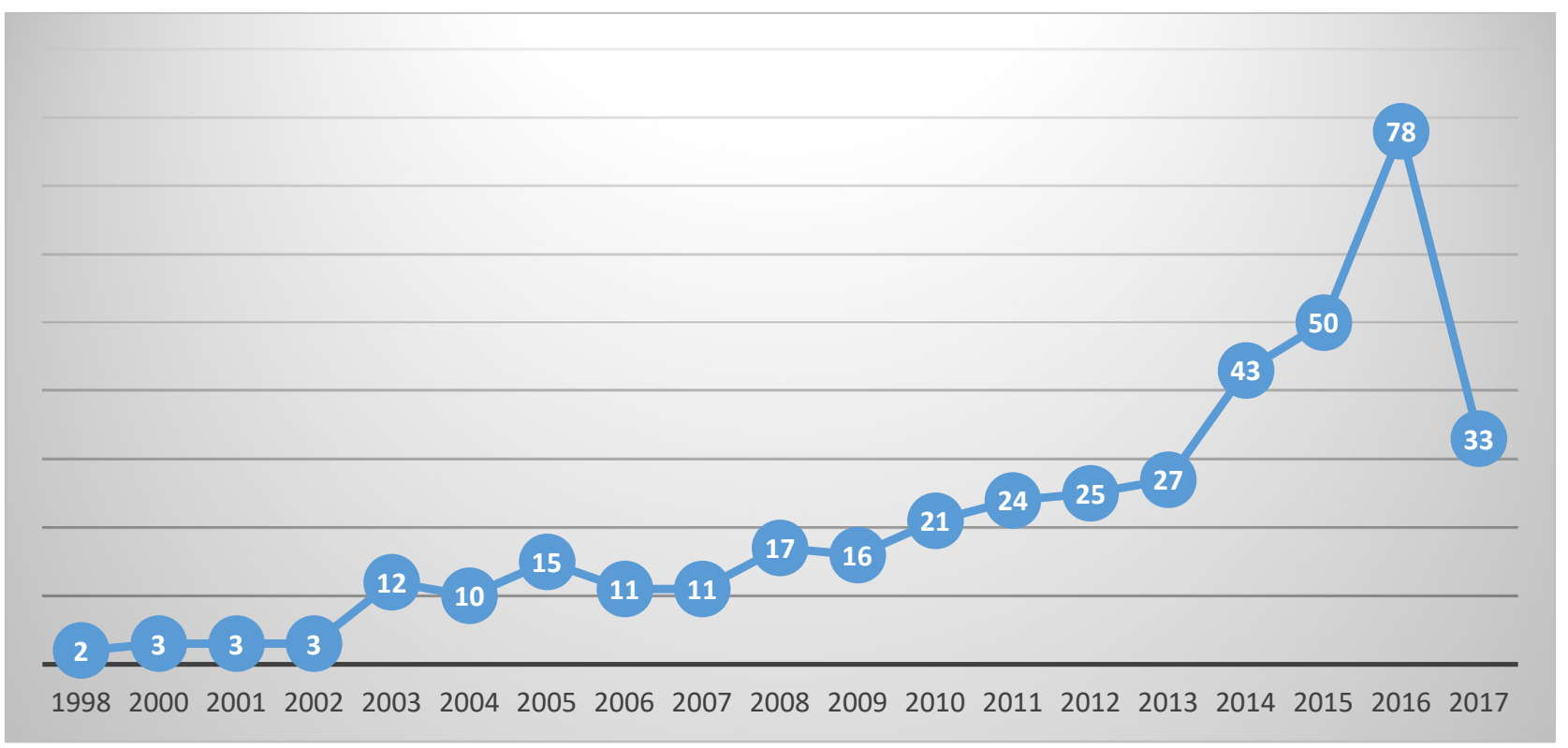

Figure 2: Number of mobile payment services research articles published annually (1997-2017)

The 25 journals that had the maximum number of references and interconnection between them have been enlisted in Figure 3. These journals together published 181 articles, which amounts to $44.5 \%$ of the total number of references obtained during the study. The Lecture Notes in Computer Science received the single highest number of references (22) followed by the Electronic Commerce Research and Applications (16), IEEE Transactions on Wireless Communications and Wireless Personal Communications (13).

Figures $4 \& 5$ depict the details of the various countries to which the most influential authors belonged. As many as 2,481 references were obtained. Geographically, the western side of the world was represented by the USA, UK, Canada and Germany coming up with 95, 28, 27 and 20 references, respectively. Asia was well-represented by China, South Korea and Taiwan with 80, 30 and 28 references, respectively. 


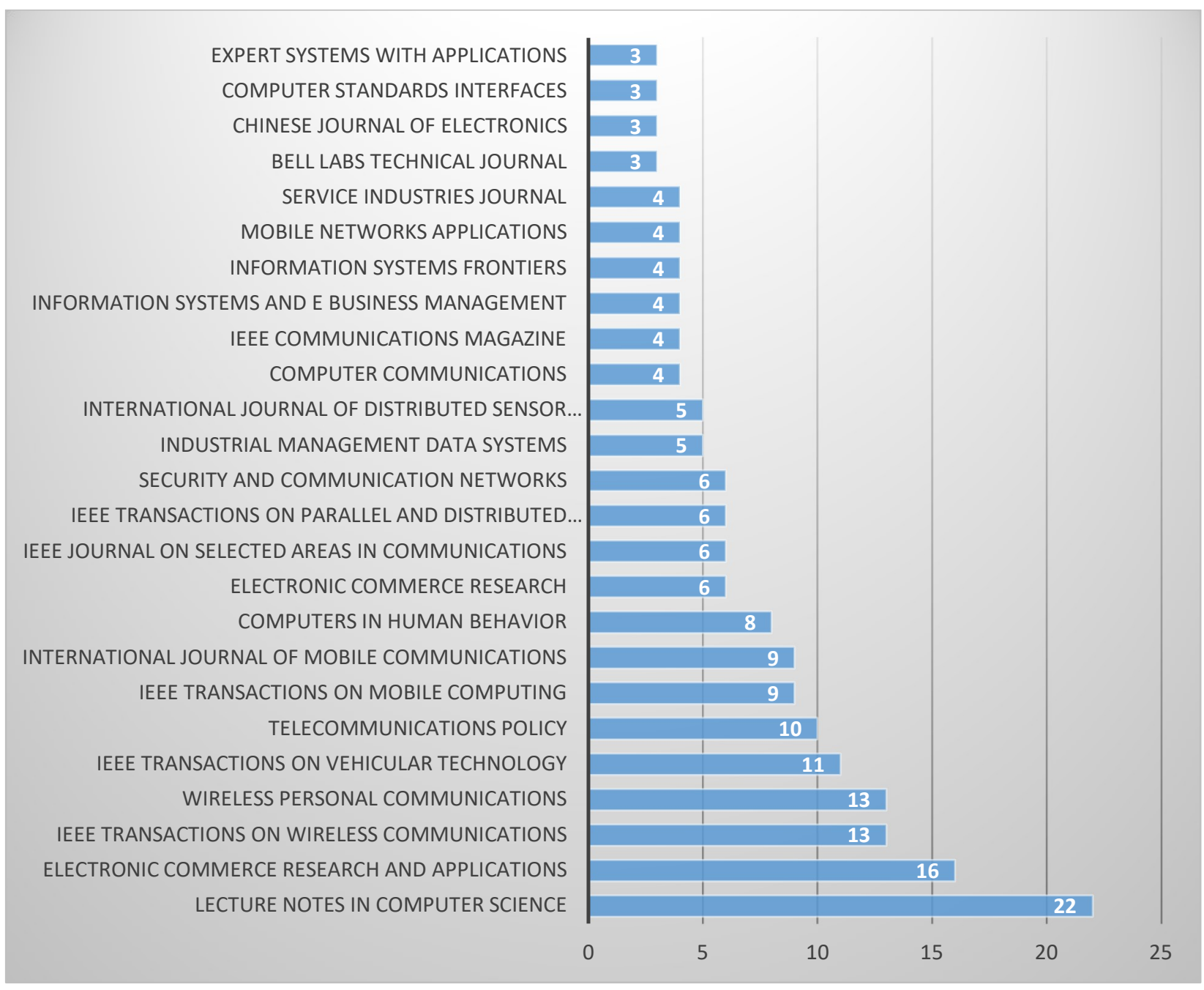

Figure 3: Distribution of source documents by journals

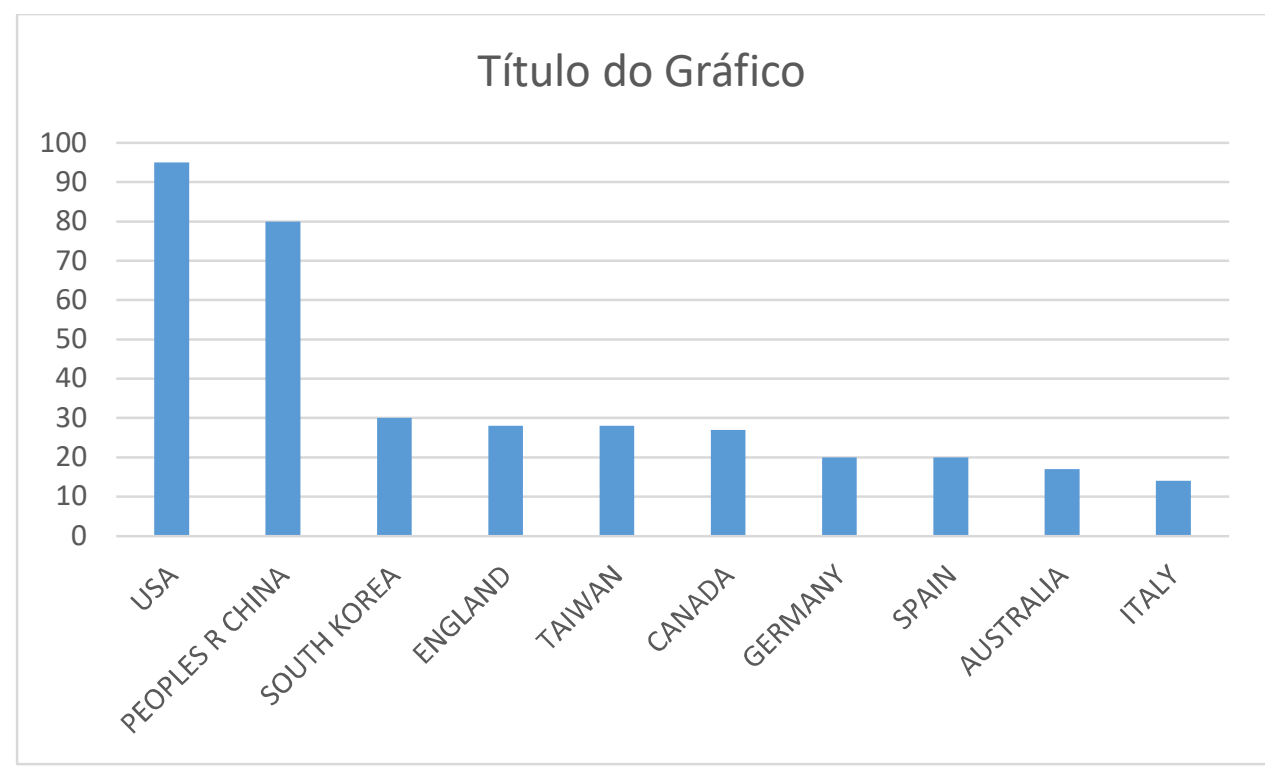

Figure 4: Number of references by country (Top 10) 


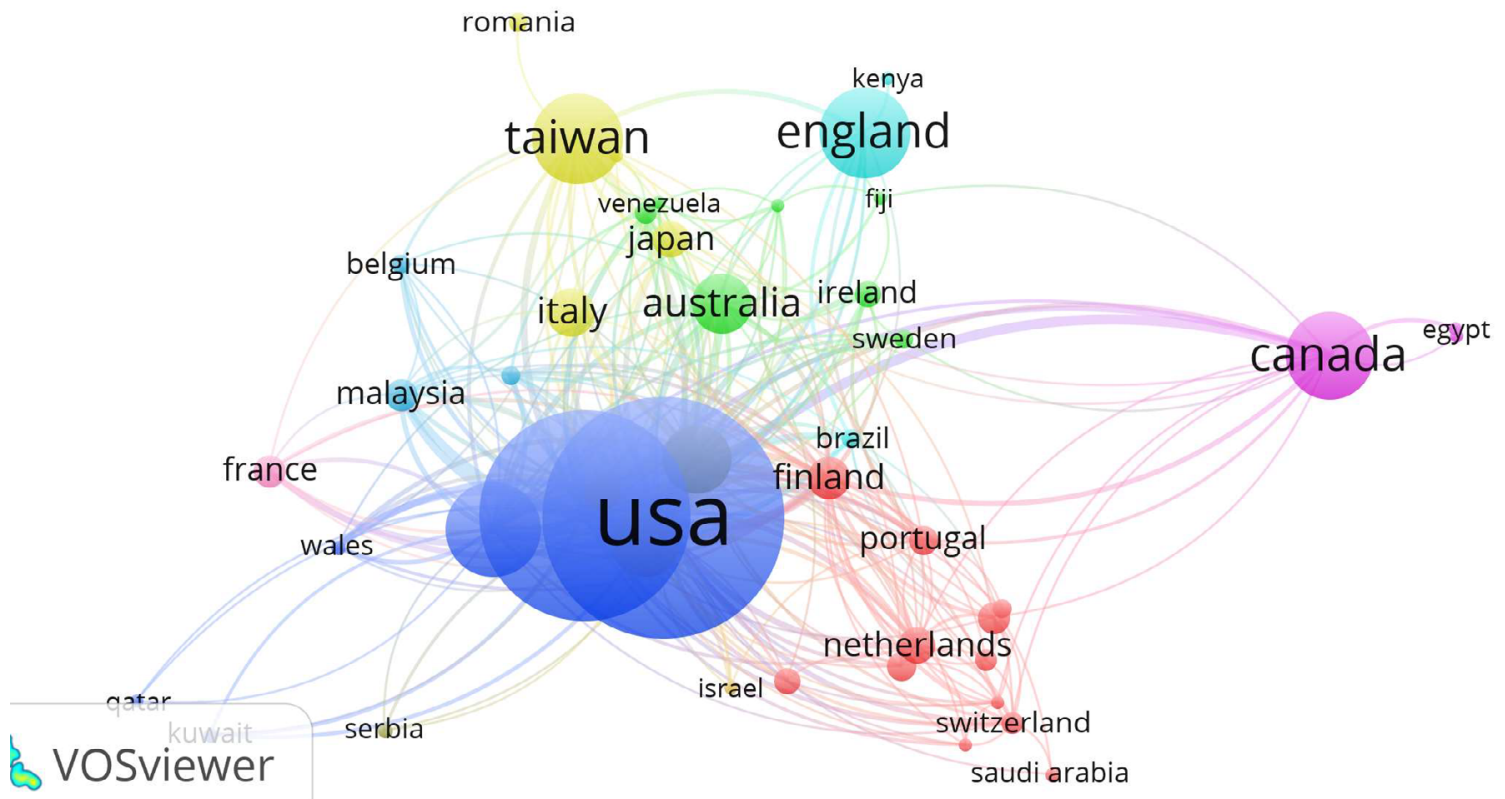

Figure 5: Interconnection between the references by country

Having established the dataset of mobile payment services research articles, the abovementioned 3-tier analytical procedure (depicted in Figure 1) was applied for understanding the intellectual structure of mobile payment services. The ensuing sub-sections have been arranged as below:

1. Citation results of the dataset have been explained through the citation analysis

2. Multivariate analysis test results have been presented via co-citation analysis

3. Network analysis tests have been given under the Social Network Analysis

\section{Citation Analysis}

As many as 406 cited research articles and 3,424 citation documents related to mobile payment services research were obtained at the end of the search process from 1997 to 2017 (Figure 6).

Figure 6 depicts the citation network prepared from the 406 articles obtained from WoK. A critical measure for ranking citation networks is Network Density. Jeong et al. (2014) define network density as 'the number of connections between nodes in a network.' Wang et al. (2016) further elucidate that for a fully connected graph, i.e., when each node in a network is connected with all the other nodes, the network density is taken to be one, whereas the score is zero if there are no connections. In other words, the quantification of network density ranges from 0-1. Abrahamson and Rosenkopf (1997) clarify that values of 0.5 and below depict low density while high density is denoted by values of 0.5 and above. It emerges from the present study that the citation relationship between the documents is not very robust. Network density value of 0.00140729 was obtained, which rose to 0.02083102 after the elimination of 266 isolated nodes. Finally, the citation network contained 140 research articles. 


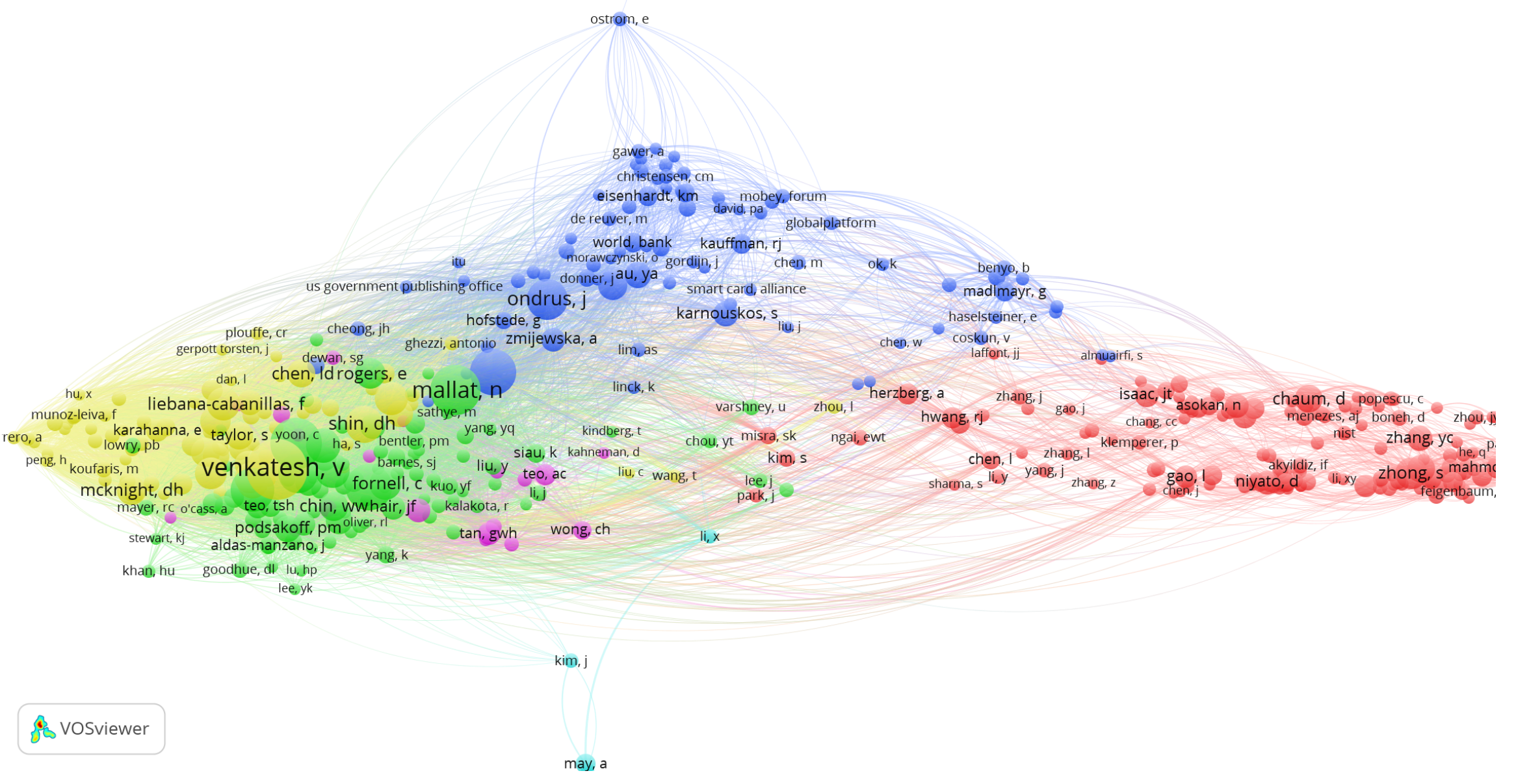

Figure 6: 406 sources of mobile payment services and citing references 


\section{Co-citation Analysis}

The 140 connected documents obtained from the citation network analysis discussed above, and the 3,424 citing documents, were used to prepare a co-citation matrix (Figures 6, $7 \& 8$ ). The cocitation matrix was normalised using Pearson's correlation coefficient prior to the statistical analysis using UCINET. Factor analysis as well as multi-dimensional scaling were used in the present study to ascertain the intellectual structure and envision the article distribution.

Principal Component Analysis (abbreviated as PCA) was employed to conduct factor analysis with Varimax (orthogonal) rotation (Hair et al., 1998). UCINET is an analytical tool which permits both eigenvalue and number of factors as the cut-off point. Based on factor analysis, this study considered only those research articles that had factor loadings values of 0.6 or greater (Hair et al., 2010) to ensure that only relevant documents were included. To start with, eigenvalues greater than 1 were set for factor analysis, whereas 10 factors were selected. 75.3 percent of the total variance was explained and 10 factors emerged at the end of this study, with factors eight, nine and ten ending with 1 item each; these were articles by Donner and Tellez (2008),Funk, (2007) and Zhong and Wu (2010), respectively. Therefore, in the next round of iteration, while the eigenvalue remained at greater than 1, the number of factors dropped to eight. Finally, seven factors remained, which were found to explain 68.62 percent of the total variance. As per Hair et al. (2010), when factors can explain more than 50 percent of the total variance, it is acceptable, while more than 70 percent is considered high. Table 1 presents the factor analysis results. Several of the references were found to attain a factor loading less than 0.6 and had to be eliminated from study. Finally, 88 articles with factor loading greater than 0.6 were considered for the further analysis.

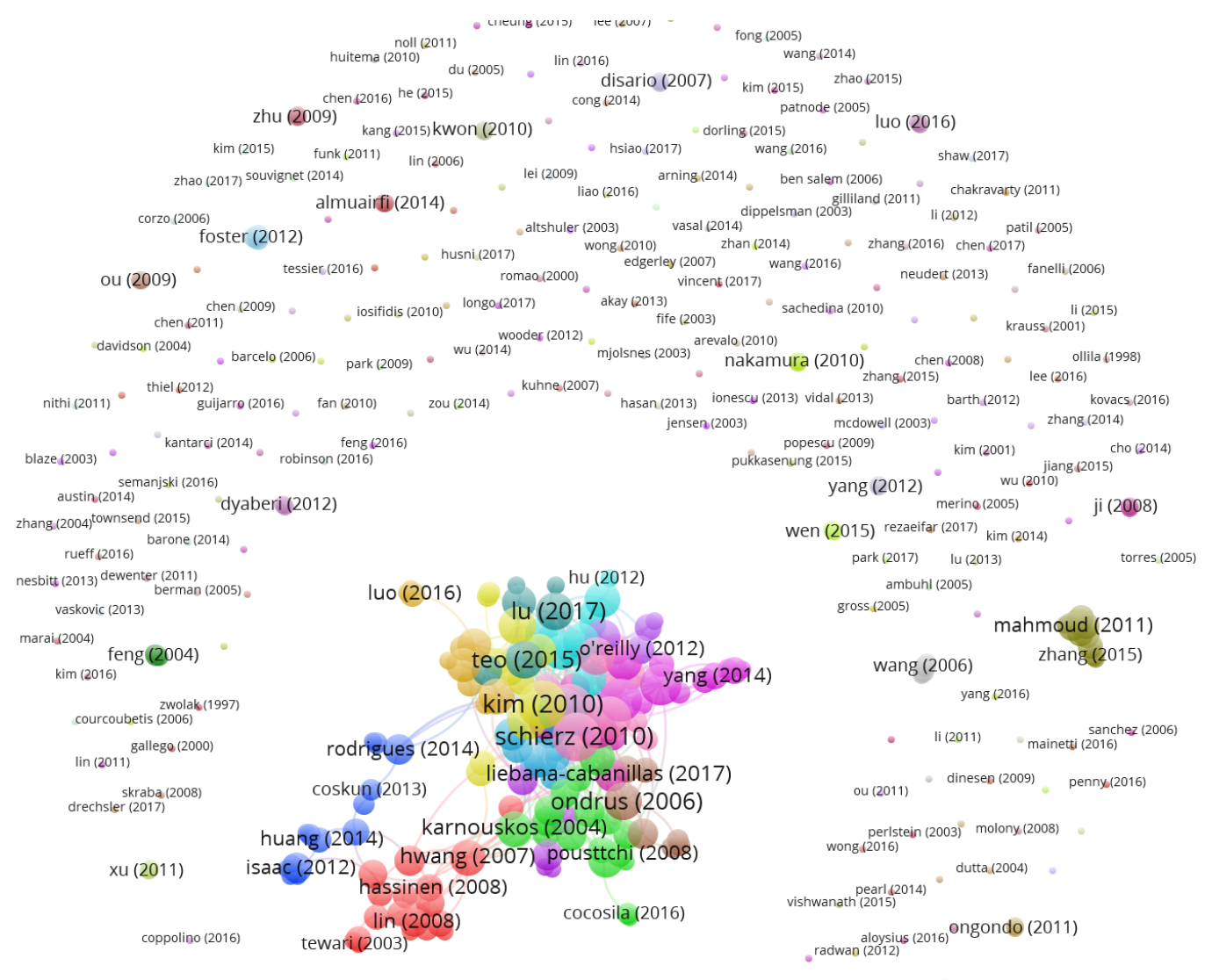

Figure 7: Citation Network of 406 mobile payment services research articles 
Factor 1 explained $36.85 \%$ of the variance and most of the factor received higher factor loading. This factor contains most of the mobile payment services adoption research articles including mobile payment services adoption intention, acceptance and usage. Multiple theories of adoption and acceptance were tested to measure intention of consumers and merchants towards mobile payment services implementation. More specifically, intention to use mobile payment services (De Luna et al., 2016; Seol et al., 2016), adoption (Zhou et al., 2010; Al-Jabri and Sohail, 2012), acceptance (Di Pietro et al., 2015), user satisfaction (Yang et al., 2016) and usage intention (Schierz et al. 2010; Sripalawat et al., 2011). Factor 2 was dominated by articles related to trust (Zhou, 2013; Shin, 2010), risk (Seol et al., 2016; Slade et al., 2015) and security (Musa et al., 2015; Oliveira et al., 2016) of mobile payment services. The variance explained by this factor was 11.33 percent. Factor 3 focused on applications of mobile payment services. Ruiz-del-Olmo et al. (2014), Cagliano et al. (2017), Gerpott and Meinert (2016), Miao and Jayakar (2016), Pousttchi et al. (2009) etc. based on these works, therefore, factor 3 was labelled as mobile payment services application. The variance explained by this was 6.21 percent. Factor 4 was dominated by articles related to scheme related to mobile payment services. The variance explained by this factor was 4.88 percent and consisted the work of Yang et al. (2013), Han et al. (2016), Martinez-Pelaez et al. (2015) and Shi et al. (2013). Factor 5 focused on mobile payment services protocol. Falahati and Jannati (2015), Issac and Zeadally (2014), Lee et al. (2008), Li et al. (2012) and Janav et al. (2014) based on protocols of mobile payment works, therefore, factor 5 was labelled as mobile payment services application. The variance explained by this was 4.11 percent. Factor 6 explained $3.59 \%$ of the variance and contains most of the mobile payment services architecture research. Factor 7 focused on mobile payment market cooperation and explained $1.66 \%$ of the variance. Hedman et al. (2015), Liu et al. (2015) and de Reuver et al. (2015) research work are based on mobile payment market cooperation.

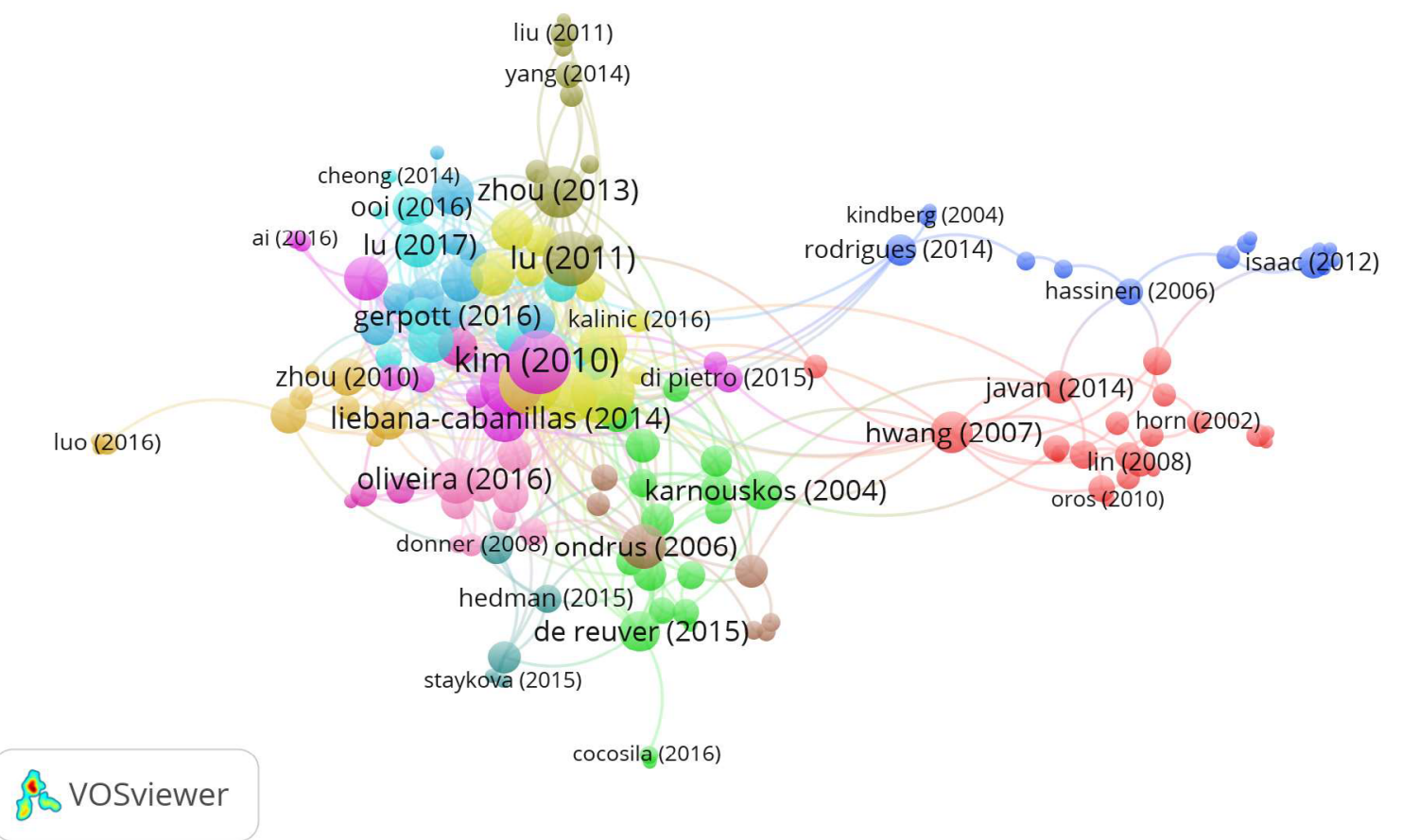

Figure 8: 140 un-isolated mobile payment research article 
The factor analysis method indicates that a research article can potentially appear in more than one factor. However, multi-dimensional scaling ensures that a document is located in only one graphical point. Hence, multi-dimensional scaling can aid factor analysis findings in positioning a document in a key sub-research domain. Finally, all 88 research articles from the present dataset were put on a network map; this has been depicted in Table 1 and Figure 9, which were limited to links having correlation coefficient values higher than 0.6 . Also, the stress value obtained was 0.102 , which is below the minimal acceptable value of 0.2. Both multi-dimensional scaling and factor analysis were in accord with respect to the grouping of research articles, and did not omit any key themes of research in the selected study area.

\section{Social Network Analysis}

Social network analysis helps visualise how research documents are connected with each other, revealing the key characteristics and extent of linkage among the documents that form a network map. Detailed investigation elucidated the effect of a few keynote research articles on the sub-domains of the selected study area. Here, social network analysis was employed via the three centrality measures outlined in the methodology section.

A summary of the significant articles in the research area of mobile payment services research articles, as per their three different centrality measures, has been provided in Table 2 . It emerges that Schierz et al., (2010) emerged as the dominating author group as per the centrality measure approach. As per degree centrality, a document authored by Schierz et al. (2010) received the largest connection in the network map. In addition, five of the other keynote articles are authored by Kim et al. (2010), Yang and Chang (2012), Chen (2008), Zhou (2013) and Liebana-Cabanillas et al. (2014). An article by (Seol et al., 2016) emerged as the lowest degree centrality scorer.

According to Abbasi et al. (2012), the betweenness centrality values show the potential to connect the veteran researchers in the field with the new researchers as the new researchers largely link to betweenness centrality. A document by Schierz et al., (2010) was the highest scorer, followed by Kim et al. (2010), Yang and Chang (2012), Hwang et al. (2007), Chen (2008) and Zhou (2013). The position of article by Zhou (2013) has dropped in betweenness measures. Remarkably, a publication authored by Hwang et al., (2007) received a higher score as per the betweenness centrality measure as compared with the first two centrality measures; thereby, this article moved upward by seven positions.

Results of an eigenvector centrality measure could transform the entire network map relationship. As per the eigenvector centrality results, the article by Schierz et al., (2010) was found to have topped, followed by Kim et al. (2010), Yang and Chang (2012), Hwang et al. (2007), Chen (2008) and Zhou (2013). An article by Al-Jabri et al. (2012), Seol et al. (2016), Ramos-de-Luna et al. (2016), Ruiz-delOlmo et al. (2014), Lee et al. (2008), Li et al. (2012), Liang and Yeh (2011), Musa et al. (2015) and Pousttchi et al. (2009) received zero score of eigenvector centrality. 
Table 1: Factor analysis of mobile payment services articles

\begin{tabular}{|c|c|c|c|c|c|c|}
\hline Factor1 & Factor2 & Factor3 & Factor4 & Factor5 & Factor6 & Factor7 \\
\hline Adoption & Trust, risk and security & Application & Scheme & Protocol & Architecture & $\begin{array}{l}\text { Mobile payment market } \\
\text { cooperation }\end{array}$ \\
\hline de Kerviler et al. (2016) & Kim et al. (2010) & $\begin{array}{l}\text { Ruiz-del-Olmo et al. } \\
\text { (2014) }\end{array}$ & Yang et al. (2014) & $\begin{array}{c}\text { Falahati and Jannati } \\
\text { (2015) }\end{array}$ & Karnouskos (2004) & Hedman et al. (2015) \\
\hline $\begin{array}{c}\text { Liebana-Cabanillas et } \\
\text { al. (2014) }\end{array}$ & Yang (2016) & Cagliano et al. (2017) & Hwang et al. (2007) & $\begin{array}{c}\text { Issac and Zeadally } \\
\text { (2014) }\end{array}$ & Wan et al. (2014) & Liu et al. (2015) \\
\hline $\begin{array}{l}\text { Liebana-Cabanillas et } \\
\text { al. (2014) (b) }\end{array}$ & Zhou (2014) & $\begin{array}{c}\text { Gerpott and Meinert } \\
\text { (2016) }\end{array}$ & Yang et al. (2013) & Lee et al. (2008) & Huang et al. (2014) & de Reuver et al. (2015) \\
\hline $\begin{array}{c}\text { Cheng and Huang } \\
\text { (2013) }\end{array}$ & Rouibah et al. (2016) & $\begin{array}{c}\text { Gerpott and Meinert } \\
\text { (2016) }\end{array}$ & $\begin{array}{c}\text { Yang and Chang (2012) } \\
\text { (b) }\end{array}$ & Li et al. (2012) & Yang and Lou (2016) & \\
\hline Duane et al. (2014) & Xin et al. (2015) & $\begin{array}{c}\text { Miao and Jayakar } \\
\text { (2016) }\end{array}$ & Han et al. (2016) & Janav et al. (2014) & & \\
\hline Teo et al. (2015) (b) & $\begin{array}{c}\text { Hillman and } \\
\text { Neustaedter (2017) }\end{array}$ & Pousttchi et al. (2009) & $\begin{array}{c}\text { Martinez-Pelaez et al. } \\
\qquad(2015)\end{array}$ & & & \\
\hline Oliveira et al. (2016) & Gao et al. (2017) & Rodrigues et al. (2014) & Shi et al. (2013) & & & \\
\hline Musa et al. (2015) & Chen and Liu (2016) & Madureira (2017) & & & & \\
\hline Karjaluoto et al. (2014) & Yang et al. (2015) & O'Reilly et al. (2012) & & & & \\
\hline Seol et al. (2016) & $\begin{array}{l}\text { Thakur and Srivastava } \\
\qquad(2014)\end{array}$ & Yang (2013) & & & & \\
\hline \multicolumn{7}{|l|}{$\begin{array}{l}\text { Koenig-Lewis et al. } \\
\text { (2015) }\end{array}$} \\
\hline \multicolumn{7}{|l|}{$\begin{array}{l}\text { Kalinic and Marinkovic } \\
\qquad(2016)\end{array}$} \\
\hline \multicolumn{7}{|l|}{$\begin{array}{l}\text { Ramos-de-Luna et al. } \\
\text { (2016) }\end{array}$} \\
\hline \multicolumn{7}{|l|}{$\begin{array}{l}\text { Khalilzadeh et al. } \\
\text { (2017) }\end{array}$} \\
\hline \multicolumn{7}{|l|}{ Moorthy et al. (2017) } \\
\hline \multicolumn{7}{|l|}{ Lu et al. (2017) } \\
\hline \multicolumn{7}{|l|}{$\begin{array}{l}\text { Madan and Yadav } \\
\qquad(2016)\end{array}$} \\
\hline \multicolumn{7}{|l|}{$\begin{array}{l}\text { Liebana-Cabanillas et } \\
\text { al. (2015) }\end{array}$} \\
\hline \multicolumn{7}{|l|}{ Zhang et al. (2012) } \\
\hline \multicolumn{7}{|l|}{ Zhou et al. (2010) } \\
\hline Zhou (2013) & & & & & & \\
\hline
\end{tabular}


Table 1: Cont.

\begin{tabular}{|c|c|c|c|c|c|c|}
\hline Factor1 & Factor2 & Factor3 & Factor4 & Factor5 & Factor6 & Factor7 \\
\hline Adoption & Trust, risk and security & Application & Scheme & Protocol & Architecture & $\begin{array}{l}\text { Mobile payment market } \\
\text { cooperation }\end{array}$ \\
\hline Liu and $\mathrm{Li}$ (2011) & & & & & & \\
\hline Liang and Yeh (2011) & & & & & & \\
\hline Al-Jabri et al. (2012) & & & & & & \\
\hline Kapoor et al. (2015) & & & & & & \\
\hline Keramati et al. (2012) & & & & & & \\
\hline $\begin{array}{l}\text { Upadhyay and Jahanyan } \\
\text { (2016) }\end{array}$ & & & & & & \\
\hline $\begin{array}{c}\text { Upadhyay and } \\
\text { Chattopadhyay (2015) }\end{array}$ & & & & & & \\
\hline Kim et al. (2010) & & & & & & \\
\hline Schierz et al. (2010) & & & & & & \\
\hline Chen (2008) & & & & & & \\
\hline Yang et al. (2012) & & & & & & \\
\hline Liu et al. (2016) & & & & & & \\
\hline Yang et al. (2017) & & & & & & \\
\hline Slade et al. (2015) & & & & & & \\
\hline Teo et al. (2015) & & & & & & \\
\hline $\begin{array}{l}\text { Liebana-Cabanillas and } \\
\text { Montoro-Rios (2015) }\end{array}$ & & & & & & \\
\hline Ooi and Tan (2016) & & & & & & \\
\hline Apanasevic et al. (2016) & & & & & & \\
\hline $\begin{array}{l}\text { Phonthanukitithaworn et } \\
\text { al. (2016) }\end{array}$ & & & & & & \\
\hline Francisco et al. (2015) & & & & & & \\
\hline Kujala et al. (2017) & & & & & & \\
\hline $\begin{array}{c}\text { Liebana-Cabanillas et } \\
\text { al. (2017) }\end{array}$ & & & & & & \\
\hline $\begin{array}{l}\text { Ramos de Luna et al. } \\
\text { (2017) }\end{array}$ & & & & & & \\
\hline $\begin{array}{l}\text { Phonthanukitithaworn et } \\
\text { al. (2016) (b) }\end{array}$ & & & & & & \\
\hline Sripalawat et al. (2011) & & & & & & \\
\hline
\end{tabular}


Table 1: Cont.

\begin{tabular}{|c|c|c|c|c|c|c|c|}
\hline & Factor1 & Factor2 & Factor3 & Factor4 & Factor5 & Factor6 & Factor7 \\
\hline & Adoption & Trust, risk and security & Application & Scheme & Protocol & Architecture & $\begin{array}{l}\text { Mobile payment market } \\
\text { cooperation }\end{array}$ \\
\hline & Duane et al. (2014) & & & & & & \\
\hline & Di Pietro et al. (2015) & & & & & & \\
\hline & Shin (2010) & & & & & & \\
\hline & Yang et al. (2014) (b) & & & & & & \\
\hline & $\begin{array}{l}\text { Molina-Castillo et al. } \\
\text { (2016) }\end{array}$ & & & & & & \\
\hline $\begin{array}{l}\% \text { of variance } \\
\text { explained }\end{array}$ & 36.85 & 11.33 & 6.21 & 4.88 & 4.11 & 3.59 & 1.66 \\
\hline $\begin{array}{l}\text { Sum of } \\
\text { Per cent of } \\
\text { variance } \\
\text { explained }\end{array}$ & 36.85 & 48.18 & 54.40 & 59.27 & 63.38 & 66.97 & 68.62 \\
\hline
\end{tabular}




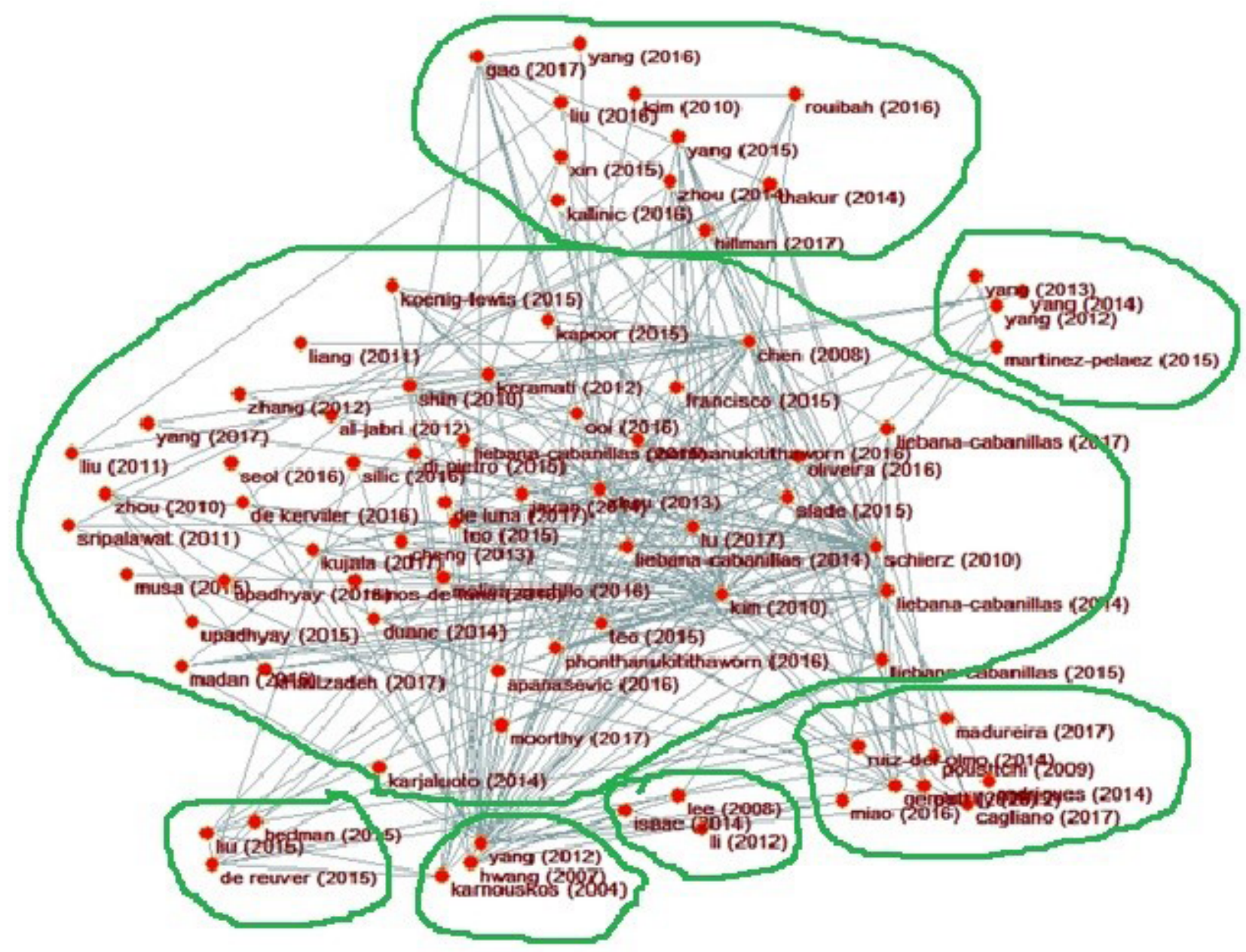

Figure 9: Social network of mobile payment services literature

Table 2: Result of Centrality Measures

\begin{tabular}{|c|c|c|c|c|c|}
\hline Authors & Degree & Authors & Eigenvalue & Authors & Betweenness \\
\hline Schierz et al. (2010) & 41 & Schierz et al. (2010) & 0.307 & Schierz et al. (2010) & 774.324 \\
\hline Kim et al. (2010) & 39 & Kim et al. (2010) & 0.306 & Kim et al. (2010) & 574.984 \\
\hline Yang and Chang (2012) & 36 & $\begin{array}{l}\text { Yang and Chang } \\
\text { (2012) }\end{array}$ & 0.299 & $\begin{array}{l}\text { Yang and Chang } \\
\text { (2012) }\end{array}$ & 425.661 \\
\hline Chen (2008) & 25 & Zhou (2013) & 0.225 & Hwang et al. (2007) & 403.492 \\
\hline Zhou (2013) & 24 & Chen (2008) & 0.222 & Chen (2008) & 277.315 \\
\hline Liebana-Cabanillas et al. (2014) & 19 & $\begin{array}{l}\text { Liebana-Cabanillas et } \\
\text { al. (2014) }\end{array}$ & 0.208 & Zhou (2013) & 271.316 \\
\hline $\begin{array}{l}\text { Liebana-Cabanillas and Montoro-Rios } \\
\text { (2015) }\end{array}$ & 15 & $\begin{array}{l}\text { Liebana-Cabanillas et } \\
\text { al. (2015) }\end{array}$ & 0.181 & $\begin{array}{l}\text { Martinez-Pelaez et al. } \\
\text { (2015) }\end{array}$ & 147.497 \\
\hline Shin (2010) & 15 & Lu et al. (2017) & 0.176 & Oliveira et al. (2016) & 91.938 \\
\hline Lu et al. (2017) & 14 & Teo et al. (2015) & 0.162 & $\begin{array}{l}\text { Molina-Castillo et al. } \\
\text { (2016) }\end{array}$ & 82.782 \\
\hline Oliveira et al. (2016) & 14 & Teo et al. (2015) (b) & 0.159 & $\begin{array}{l}\text { Issac and Zeadally } \\
\text { (2014) }\end{array}$ & 79 \\
\hline
\end{tabular}


Table 2: Cont.

\begin{tabular}{|c|c|c|c|c|c|}
\hline Authors & Degree & Authors & Eigenvalue & Authors & Betweenness \\
\hline Teo et al. (2015) & 14 & $\begin{array}{c}\text { Liebana-Cabanillas } \\
\text { and Montoro-Rios } \\
(2015)\end{array}$ & 0.158 & $\begin{array}{c}\text { Thakur and Srivastava } \\
\text { (2014) }\end{array}$ & 75.009 \\
\hline Hwang et al. (2007) & 13 & Slade et al. (2015) & 0.158 & Yang et al. (2013) & 64.485 \\
\hline Liebana-Cabanillas et al. (2015) & 13 & Shin (2010) & 0.149 & Yang et al. (2017) & 62.984 \\
\hline Slade et al. (2015) & 13 & $\begin{array}{l}\text { Phonthanukitithaworn } \\
\text { et al. (2016) (b) }\end{array}$ & 0.147 & de Reuver et al. (2015) & 62.544 \\
\hline Teo et al. (2015) (b) & 13 & $\begin{array}{l}\text { Koenig-Lewis et al. } \\
\text { (2015) }\end{array}$ & 0.146 & $\begin{array}{c}\text { Liebana-Cabanillas } \\
\text { and Montoro-Rios } \\
(2015)\end{array}$ & 58.345 \\
\hline Phonthanukitithaworn et al. (2016) (b) & 12 & $\begin{array}{l}\text { Upadhyay and } \\
\text { Jahanyan (2016) }\end{array}$ & 0.138 & Yang et al. (2014) & 55.927 \\
\hline Thakur and Srivastava (2014) & 12 & Cagliano et al. (2017) & 0.137 & $\begin{array}{c}\text { Liebana-Cabanillas et } \\
\text { al. (2014) }\end{array}$ & 54.093 \\
\hline Upadhyay and Jahanyan (2016) & 12 & Oliveira et al. (2016) & 0.136 & $\begin{array}{l}\text { Phonthanukitithaworn } \\
\text { et al. (2016) (b) }\end{array}$ & 53.42 \\
\hline Yang et al. (2015) & 12 & Yang et al. (2015) & 0.136 & Di Pietro et al. (2015) & 53.305 \\
\hline Yang et al. (2017) & 11 & Chen and Liu (2016) & 0.135 & Teo et al. (2015) (b) & 52.643 \\
\hline Cagliano et al. (2017) & 10 & Kujala et al. (2017) & 0.127 & Lu et al. (2017) & 50.765 \\
\hline Chen and Liu (2016) & 10 & Gao et al. (2017) & 0.124 & $\begin{array}{l}\text { Liebana-Cabanillas et } \\
\text { al. (2014) (b) }\end{array}$ & 47.868 \\
\hline Gao et al. (2017) & 10 & Yang et al. (2017) & 0.12 & $\begin{array}{l}\text { Upadhyay and } \\
\text { Jahanyan (2016) }\end{array}$ & 44.753 \\
\hline Gerpott and Meinert (2016) & 10 & $\begin{array}{c}\text { Gerpott and Meinert } \\
\text { (2016) }\end{array}$ & 0.118 & Shin (2010) & 43.976 \\
\hline Koenig-Lewis et al. (2015) & 10 & $\begin{array}{l}\text { Liebana-Cabanillas et } \\
\text { al. (2014) (b) }\end{array}$ & 0.117 & Rouibah et al. (2016) & 43.064 \\
\hline Kujala et al. (2017) & 10 & Francisco et al. (2015) & 0.115 & Teo et al. (2015) & 38.004 \\
\hline Liebana-Cabanillas et al. (2014) (b) & 10 & $\begin{array}{c}\text { Liebana-Cabanillas et } \\
\text { al. (2017) }\end{array}$ & 0.113 & Zhou et al. (2010) & 34.19 \\
\hline Zhou et al. (2010) & 10 & $\begin{array}{c}\text { de Reuver et al. } \\
\text { (2015) }\end{array}$ & 0.112 & Chen and Liu (2016) & 31.352 \\
\hline de Reuver et al. (2015) & 9 & $\begin{array}{l}\text { Phonthanukitithaworn } \\
\text { et al. (2016) }\end{array}$ & 0.111 & Liu et al. (2015) & 31.325 \\
\hline Martinez-Pelaez et al. (2015) & 9 & $\begin{array}{c}\text { Martinez-Pelaez et al. } \\
\text { (2015) }\end{array}$ & 0.107 & Gao et al. (2017) & 31.263 \\
\hline Di Pietro et al. (2015) & 8 & Zhou (2014) & 0.098 & $\begin{array}{c}\text { Gerpott and Meinert } \\
\text { (2016) }\end{array}$ & 30.923 \\
\hline Francisco et al. (2015) & 8 & Ooi and Tan (2016) & 0.096 & $\begin{array}{c}\text { Upadhyay and } \\
\text { Chattopadhyay (2015) }\end{array}$ & 28.533 \\
\hline Phonthanukitithaworn et al. (2016) & 8 & $\begin{array}{l}\text { Thakur and Srivastava } \\
\text { (2014) }\end{array}$ & 0.095 & Slade et al. (2015) & 28.269 \\
\hline Zhou (2014) & 8 & $\begin{array}{c}\text { Apanasevic et al. } \\
\text { (2016) }\end{array}$ & 0.093 & Liu et al. (2016) & 22.525 \\
\hline Apanasevic et al. (2016) & 7 & Moorthy et al. (2017) & 0.082 & $\begin{array}{c}\text { Phonthanukitithaworn } \\
\text { et al. (2016) }\end{array}$ & 21.911 \\
\hline Karnouskos (2004) & 7 & $\begin{array}{c}\text { Madan and Yadav } \\
\text { (2016) }\end{array}$ & 0.081 & Duane et al. (2014) & 20.151 \\
\hline Liebana-Cabanillas et al. (2017) & 7 & Duane et al. (2014) & 0.075 & Yang et al. (2015) & 19.507 \\
\hline Moorthy et al. (2017) & 7 & Zhou et al. (2010) & 0.073 & Janav et al. (2014) & 18.027 \\
\hline
\end{tabular}


Table 2: Cont.

\begin{tabular}{|c|c|c|c|c|c|}
\hline Authors & Degree & Authors & Eigenvalue & Authors & Betweenness \\
\hline Duane et al. (2014) & 6 & Di Pietro et al. (2015) & 0.067 & Moorthy et al. (2017) & 17.463 \\
\hline Liu et al. (2015) & 6 & Kapoor et al. (2015) & 0.066 & Karnouskos (2004) & 15.415 \\
\hline Ooi and Tan (2016) & 6 & $\begin{array}{c}\text { de Kerviler et al. } \\
\text { (2016) }\end{array}$ & 0.063 & Yang et al. (2012) & 14.695 \\
\hline Janav et al. (2014) & 5 & Karnouskos (2004) & 0.06 & $\begin{array}{c}\text { Liebana-Cabanillas et } \\
\text { al. (2015) }\end{array}$ & 13.609 \\
\hline Kapoor et al. (2015) & 5 & $\begin{array}{l}\text { Khalilzadeh et al. } \\
\text { (2017) }\end{array}$ & 0.06 & $\begin{array}{c}\text { Apanasevic et al. } \\
\text { (2016) }\end{array}$ & 13.413 \\
\hline Khalilzadeh et al. (2017) & 5 & $\begin{array}{c}\text { Hillman and } \\
\text { Neustaedter (2017) }\end{array}$ & 0.059 & Kujala et al. (2017) & 12.004 \\
\hline Madan and Yadav (2016) & 5 & Rouibah et al. (2016) & 0.058 & $\begin{array}{l}\text { Khalilzadeh et al. } \\
\text { (2017) }\end{array}$ & 11.971 \\
\hline Upadhyay and Chattopadhyay (2015) & 5 & $\begin{array}{c}\text { Kalinic and } \\
\text { Marinkovic (2016) }\end{array}$ & 0.057 & Cagliano et al. (2017) & 10.64 \\
\hline de Kerviler et al. (2016) & 4 & Hwang et al. (2007) & 0.051 & Zhou (2014) & 8.999 \\
\hline Duane et al. (2014) & 4 & Slice and Back (2016) & 0.047 & Francisco et al. (2015) & 8.898 \\
\hline Hillman and Neustaedter (2017) & 4 & $\begin{array}{l}\text { Miao and Jayakar } \\
\quad(2016)\end{array}$ & 0.046 & $\begin{array}{l}\text { Koenig-Lewis et al. } \\
\qquad(2015)\end{array}$ & 7.939 \\
\hline Keramati et al. (2012) & 4 & O’Reilly et al. (2012) & 0.046 & Kim et al. (2010) & 7.533 \\
\hline Madureira (2017) & 4 & $\begin{array}{c}\text { Cheng and Huang } \\
\text { (2013) }\end{array}$ & 0.045 & $\begin{array}{c}\text { Miao and Jayakar } \\
\text { (2016) }\end{array}$ & 7.319 \\
\hline Molina-Castillo et al. (2016) & 4 & Liu et al. (2015) & 0.043 & $\begin{array}{c}\text { Liebana-Cabanillas et } \\
\text { al. (2017) }\end{array}$ & 6.108 \\
\hline Rouibah et al. (2016) & 4 & $\begin{array}{l}\text { Ruiz-del-Olmo et al. } \\
\qquad \text { (2014) }\end{array}$ & 0.042 & Madureira (2017) & 5.8 \\
\hline Sripalawat et al. (2011) & 4 & Duane et al. (2014) & 0.039 & Duane et al. (2014) & 4.88 \\
\hline Xin et al. (2015) & 4 & Janav et al. (2014) & 0.038 & Xin et al. (2015) & 4.495 \\
\hline Yang et al. (2014) & 4 & $\begin{array}{l}\text { Rodrigues et al. } \\
\text { (2014) }\end{array}$ & 0.037 & $\begin{array}{c}\text { Sripalawat et al. } \\
\text { (2011) }\end{array}$ & 3.431 \\
\hline Yang et al. (2013) & 4 & Yang and Lou (2016) & 0.034 & Ooi and Tan (2016) & 3.266 \\
\hline Cheng and Huang (2013) & 3 & Yang et al. (2014) & 0.032 & $\begin{array}{c}\text { Hillman and } \\
\text { Neustaedter (2017) }\end{array}$ & 2.861 \\
\hline Hedman et al. (2015) & 3 & Keramati et al. (2012) & 0.029 & $\begin{array}{c}\text { Madan and Yadav } \\
\text { (2016) }\end{array}$ & 2.619 \\
\hline Kalinic and Marinkovic (2016) & 3 & $\begin{array}{c}\text { Molina-Castillo et al. } \\
\text { (2016) }\end{array}$ & 0.029 & Kapoor et al. (2015) & 2.557 \\
\hline Miao and Jayakar (2016) & 3 & Xin et al. (2015) & 0.029 & Keramati et al. (2012) & 2.387 \\
\hline O’Reilly et al. (2012) & 3 & $\begin{array}{c}\text { Sripalawat et al. } \\
\text { (2011) }\end{array}$ & 0.028 & Slice and Back (2016) & 1.976 \\
\hline Slice and Back (2016) & 3 & $\begin{array}{l}\text { Karjaluoto et al. } \\
\text { (2014) }\end{array}$ & 0.027 & $\begin{array}{l}\text { Cheng and Huang } \\
\text { (2013) }\end{array}$ & 1.522 \\
\hline Yang et al. (2012) & 3 & Madureira (2017) & 0.026 & Hedman et al. (2015) & 1.332 \\
\hline Yang and Lou (2016) & 3 & $\begin{array}{l}\text { Ramos-de-Luna et al. } \\
\text { (2016) }\end{array}$ & 0.025 & $\begin{array}{l}\text { de Kerviler et al. } \\
\text { (2016) }\end{array}$ & 0.622 \\
\hline Issac and Zeadally (2014) & 2 & Musa et al. (2015) & 0.021 & $\begin{array}{l}\text { Karjaluoto et al. } \\
\text { (2014) }\end{array}$ & 0.539 \\
\hline Karjaluoto et al. (2014) & 2 & Seol et al. (2016) & 0.021 & Liu and $\operatorname{Li}(2011)$ & 0.5 \\
\hline Kim et al. (2010) & 2 & $\begin{array}{c}\text { Upadhyay and } \\
\text { Chattopadhyay (2015) }\end{array}$ & 0.019 & Yang et al. (2014) & 0.5 \\
\hline Lee et al. (2008) & 2 & Liu et al. (2016) & 0.016 & Rodrigues et al. (2014) & 0.45 \\
\hline Liu and Li (2011) & 2 & Al-Jabri et al. (2012) & 0.015 & O'Reilly et al. (2012) & 0.429 \\
\hline
\end{tabular}


Table 2: Cont.

\begin{tabular}{lccccc}
\hline Authors & Degree & Authors & Eigenvalue & Authors & Betweenness \\
\hline Liu et al. (2016) & 2 & Liang and Yeh (2011) & 0.015 & $\begin{array}{c}\text { Kalinic and } \\
\text { Marinkovic (2016) }\end{array}$ & 0.26 \\
Ramos-de-Luna et al. (2016) & 2 & Hedman et al. (2015) & 0.014 & Yang and Lou (2016) & 0.1 \\
Rodrigues et al. (2014) & 2 & Yang et al. (2013) & 0.012 & Al-Jabri et al. (2012) & 0 \\
Ruiz-del-Olmo et al. (2014) & 2 & Kim et al. (2010) & 0.008 & Seol et al. (2016) & 0 \\
Yang et al. (2014) & 2 & Issac and Zeadally & 0.004 & Ramos-de-Luna et al. & (2016) \\
Al-Jabri et al. (2012) & 1 & Lee et al. (2008) & 0.004 & Ruiz-del-Olmo et al. & (2014) \\
Li et al. (2012) & 1 & Yang et al. (2012) & 0.004 & Lee et al. (2008) & 0 \\
Liang and Yeh (2011) & 1 & Liu and Li (2011) & 0.003 & Li et al. (2012) & 0 \\
Musa et al. (2015) & 1 & Pousttchi et al. (2009) & 0.002 & Liang and Yeh (2011) & 0 \\
Pousttchi et al. (2009) & 1 & Yang et al. (2014) & 0.001 & Musa et al. (2015) & 0 \\
Seol et al. (2016) & 1 & Li et al. (2012) & 0 & Pousttchi et al. (2009) & 0 \\
\hline
\end{tabular}

\section{DISCUSSION}

The present study aimed at garnering an improved understanding of the intellectual structure of the various research publications in the field of mobile payment research. Another objective was to pinpoint those research works that had most strongly impacted the developments in this field. Factor analysis and multi-dimensional analysis were the statistical approaches used to refine the findings. Seven major themes emerged at the end of the study. These include (1) Adoption and usage; (2) Trust, risk and security; (3) Application; (4) Scheme; (5) Protocol; (6) Architecture; and (7) Mobile payment corporation.

\section{Factor 1: Adoption and usage theories/models}

This first cluster of mobile payment services research cover the period from 1997 to 2017 and underscores themes related to the way mobile payment services have been espoused world over. These articles scrutinised the determinants of mobile payment services adoption, acceptance, implementation and usage intention by consumers. Appendix summarises the adoption theories and models used by different authors to study mobile payment services adoption intention. Enormous benefits, for consumers as well as organisations, can emerge from information technology; nevertheless, user acceptance is the critical factor that determines how these benefits will be utilized. Through the review of literature it was found that, the most prominent theories/models to study the adoption and acceptance intentions like Theory of Reasoned Action (TRA) (Fishbein and Ajzen, 1975), Technology Acceptance Model (TAM) (Davis, 1989), Theory of Planned Behaviour (TPB) (Ajzen, 1991), Unified Theory of Acceptance and Use of Technology (UTAUT) (Venkatesh et al., 2003), and Diffusion of Innovation (DOI) (Rogers, 2003) among others. These models/theories were mostly applied by different authors to quantify the many perspectives and variables pertaining to mobile payment services, which included trust, usage intention, user satisfaction and user acceptance. Appendix depicts the variables that is used by different researchers to understand the adoption and acceptance of mobile payment services. Trust, Risk and Security were found to be the critical factors getting targeted by mobile payment services adoption-related publication, as more than half the studies in this cluster were aimed at investigating these variables. With the advance of digital handheld devices, 
mobile internet and applications availability, making the mobile payment services acceptable to the users has become a high priority issue. For example, the TAM and UTAUT are widely used in different tasks (Thakur and Srivastava, 2014), and the application of technology is investigated at different levels (Kalinic and Marinkovic, 2016). Mobile payment services adoption studies through TAM have accrued enormous volumes of knowledge by integrating constructs into the TAM, reflecting selfefficacy, innovation at the individual level, social influence, effort expectancy, and subjective norms among others (Appendix). For understanding the adoption of mobile payment services, the UTAUT model is emerged a useful tool which allow practitioners and business managers to comprehend the driving factors of acceptance and assess the probability that technologies of mobile payment services can be effectively taken up by consumers.

\section{Factor 2: Trust, risk and security}

Trust is an extent to measure the belief of the trustor that the trustee will fulfil the trustor's expectations without taking advantage of the trustor's vulnerabilities (Mayer et al., 1995). While adopting mobile payment services, consumers may face several operational and environmental uncertainties and thus, requires to rely on trust to overcome the risk and security perceptions. For instance, according to Hillman and Neustaedter (2017), people have trust concerns developed related to information access, data transmission and fragmented payment solutions through mobile payment. Trust mechanisms alleviate trust concerns among users for mobile shopping but did not circumvent them for mobile payments. When mobile payment services are used within physical stores, trust issues emerge along with pre-purchase anxiety and mental model challenges. The transfer based cues including trust in web shopping services and functional consistency and performance-based cues including mobile information quality and mobile service quality, significantly affect initial trust in mobile shopping services (Yang, 2015). Perceived financial risk, privacy risk, performance risk, perceived technological uncertainty, perceived information asymmetry, perceived service intangibility and perceived regulatory uncertainty were important determinant which impacts the perceived value as well as acceptance intention of mobile payment by consumers (Yang et al., 2015).

According to Chen and Liu (2016), the risks involved in mobile commerce includes: the decline in the average revenue per user caused by the price war among the mobile network operators; the lack of $3 \mathrm{G}$ consumption capacity; the huge investment in $3 \mathrm{G}$ infrastructure construction; the stability of the government communications policy; the irregular action of mobile service providers; the escalating trend of the user growth rate; the concern for user privacy; the harmonization of business recombination and acculturation; dissension over profit distribution with equipment provider; internet security risk. Consumers' willingness to adopt mobile payment depends on their assessment of the trustworthiness of mobile service vendor and provider (Xin et al., 2015). It also depends upon consumers' assessment of the functional reliability of mobile payment systems and their general disposition to trust and cultural background, specially, uncertainty avoidance (Xin et al., 2015).

Mobile service providers should adopt legal and technological structures such as third-party certifications to ensure payment security and need to continuously improve mechanisms for ensuring the integrity of personal information (Thakur and Srivastava, 2014). They also recommended to mobile service providers to engender users' innovativeness to facilitate usage of mobile payments. Rouibah et al. (2016) recommended to leverage intrinsic motivations like enjoyment and personal innovativeness and extrinsic motivation like enjoyable and gamification to understand the consumer motivation to improve the propensity of trust in electronic payment systems. Furthermore, inconvenience consumers 
experience in the transaction procedures might degrade consumers' valuation of the security and the trustworthiness of the mobile payment system (Kim et al., 2010). Initial trust plays an important role overcome unknown risks related to operational and environmental uncertainties (Gao and Waechter, 2015). Perceived information quality, perceived system quality, and perceived service quality are facilitators of initial trust formation, while perceived uncertainty is inhibitor of initial trust. Due to high perceived risk leads to decrease the initial trust associated with mobile payment adoption and usage. According to Zhou (2014), self-perception-based factors (such as, ubiquitous connection and effort expectancy) and transference-based factors (such as, structural assurance and trust in online payment) influence initial trust, which further affects performance expectancy and usage intention of mobile payment systems.

\section{Factor 3: Mobile payment services application}

Yang (2013) developed a Tobit model to analyse the contract duration phenomena which is better than churn-out information to get more information on marketing and regulation. This model also estimates the effectiveness of individual bundled components on contract duration

Ruiz Del Olmo and Belmonte-Jiménez (2014) studied the influence of the economic crisis in the methods and channels of traditional commercial communication and advertising. According to Ruiz Del Olmo and Belmonte-Jiménez (2014) usage of new media such as the telephone or mobile device, both closely linked to private, personal or intimate usage, highlights the breakdown of the traditional mass media advertising model like radio and television. Madureira (2017) developed a framework to identify the market and non-market factors affecting the success of SIM-based mobile NFC service deployment. Important obstacles for NFC includes lack of trusted service manager interoperability, interdependencies, governance and leadership, trust, NFC standardization and adoption of NFC phones, government intervention, low network effects, low competitive pressure, antitrust regulation between mobile network operators, Culture of no-collaboration between mobile network operators and more. According to Gerpott and Meinert (2016) male customers who paid through mobile carrier billing have shorter mobile network operators tenure, have higher mobile service spending, generate more mobile Internet traffic, send more SMS and use a smartphone whose OS manufacturer offers mobile carrier billing payment on its own online market place. Rodrigues et al. (2014) developed an open ecosystem that integrates different stakeholders, like customers, mobile network operators, merchants, banks, payment processors, and other technology providers and validated the application model through the deployment of two reference applications. According to O'Reilly (2012), in order to increase consumers' willingness to make mobile payments using smartphones, commercial entities need to communicate to consumers that they implement policies and employ the latest technologies to protect the privacy and data of consumers. In addition, to improve consumers' perceptions and increase their trust, regulatory bodies required to have sufficient powers to take actions against mobile service providers who do not adhere to regulatory frameworks.

\section{Factor 4: Mobile payment services schemes}

In order to improve the trust in mobile payment services, it is important to authenticate and protect the secret information transmitted between a sender and a receiver on internet. Mobile payment schemes must offer the option to authenticate each participate to prevent the participation of illegal participant. The three important participants of mobile payment services schemes includes customer, merchant and bank and consists of four phases including withdrawal, purchase, payment and deposit. 
The main security requirements for mobile payment schemes are integrity, confidentiality, mutual authentication and anonymity. The mobile payment schemes should also require low computational power, low storage capacity and low administrative cost. Researchers proposed several mobile payment services schemes to overcome these challenges. For instance, the proposed mobile payment scheme by Yang and Chang (2012) was a low computation-cost electronic payment based on the concept of the self-verified digital signature that could be efficiently applied to a large amount of mobile users without maintaining a large authentication table. This scheme using elliptic curve cryptography to reduce computation loads for large-scale user environments in mobile commerce. Opportunistic spectrum sharing approach is better than non-sharing method in performance including revenue, accept number and utilization ration in order to benefits both physical wireless networks as well as virtual networks because it decreases the price that virtual networks need to pay (Yanag et al., 2014).

Hwang et al. (2007) proposed a new on-line payment scheme for mobile network in the home as well as visited domain in order to make transaction with vendor machine, shop and WAP site based and provide consumer anonymity, data integration, non-repudiation and authentication through symmetric encryption and one-way hash function. Yang et al. (2013) proposed this mobile payment scheme based on elliptic curve cryptography that provides security like authenticity, integrity, confidentiality, privacy protection and double-spending prevention without any digital signature. In the scheme proposed by Han et al. (2016), the transactions between a buyer s and a vendor are encrypted and a mobile phone number can be a user's identity, therefore, it is not necessary to check the public key certificates. In case of a dispute, an offline adjudicator can identify dishonest party by checking the encrypted transactions (Han et al. 2016). The P2PM scheme proposed by Marti'nezPela'ez et al. (2015) have a wireless public key infrastructure with an efficient certificate path validation to provide security against well-known attacks and reduce the processing time. Shi et al. (2013) provided a countermeasure named bi-directional wormhole location mechanism to solve the colluding wormhole attack.

\section{Factor 5: Mobile payment protocol}

Mobile payment service protocols must offer robust security in order to send the financial data over wireless networks. This could be achieved by providing mutual authentication, payment authorization, confidentiality, integrity and non-repudiation between customers and merchants. Falahati and Jannati (2014) proposed a distance bounding protocol to prevent sensor networks against wormhole attack and also safeguard RFID systems against relay attack by the round trip time measurements of the executed messages. Isaac and Zeadally (2013) proposed a secure payment protocol based on the payment gateway centric scenario for mobile environments where the client cannot communicate directly with the merchant to process the payment request. Isaac and Zeadally (2013) proposed payment protocol uses symmetric-key operations with low computational requirements due to lack of direct communication between the client and the merchant. Lee et al. (2007) proposed an authentication protocol to improve the security mechanism by providing a lightweight security mechanism for mobile commerce without adopting the public key cryptosystem. Li et al. (2012) proposed an electronic payment protocol for data volume transaction in a restricted connectivity scenario of value-added applications in vehicular ad hoc network (VANET) which applied selfcertified key agreement and the vehicle could not directly communicate with the bank. The protocol proposed by Li et al. (2012) employs self-certified key agreement at reduced communication and computation cost to establish the shared key between two participants without additional message 
exchanges for efficient symmetric encryption. Javan and Bafghi (2014) proposed a payment protocol based on secure wireless payment protocol (SWPP) that provide anonymity and privacy of the customer by using a blindly signed pseudo digital certificate and anonymous bank account.

\section{Factor 6: Mobile payment architecture}

The mobile payment architecture consists of different entities are summarized in Table 3. Karnouskos (2004) discussed the mobile payment procedures and consortia required for the development of mobile payment services. According to Karnouskos (2004), a typical mobile payment scenario consists of a customer (party that make the payment), the merchant (party that accept the payment), acquirer (third party that has a relationship and interacts with the merchant) and issuer (a third party that has a relationship and interacts with the customer). The other main player of mobile payment includes the financial sector institutions (e.g. banks, credit card companies, payment processors), the mobile network operators, the government (legislation and regulation constraints) and the device. The mobile payment model like Acquirer-Centric vs. Issuer-Centric and Bank-Centric vs. Mobile Network Operators-Centric requires cooperation and coexistence of the main players for successful mobile payments. Wan et al. (2014) proposed a business model based on platform production services by integrating equipment supplier with the third-party corporations to provide comfortable, convenient and effective services. The main elements of platform production services business model includes digital platform, production and services. According to Wan et al. (2014), it important to implement internet of things sensing framework as well as inter-cloud computing architecture with platform production services business model to improve efficiency, convenience and safety in vehicular networking applications.

Huang et al. (2014) presents an overview on a third-party payment systems named BulaPay which supports secure, flexible, reliable, and efficient payment transactions to consumers and merchants for a business process in a worldwide way. The main element of a BulaPay system consists of a customer, an e-shopper (e.g. mobile phone recharge voucher sellers and an online music providers) and a BulaPay core server. The key steps of the BulaPay system includes: Build a summary payment in shopping cart; Lodge a payment request to BulaPay; Respond a Topayid and Checkout Token; Make a payment; Notify delivery; Deliver goods and services; Confirm receipt; Transfer payment. Yang and Lou (2016) developed a contracted-ruled framework for deployment and evolution of a comprehensive treatment of quality of service guarantee for practical mobile streaming markets. According to Yang and Lou (2016), the main parties involved in peer-assisted mobile peer-to-peer streaming system are Service Provider, End User and Assisting Peers. These parties are organized with contractual constraints to achieve a stable and guaranteed quality of service output. Rodrigues et al. (2014) developed an integrated mobile service solution called MobiPag, open architecture can be instantiated into a specific and complete solution for mobile payments based on NFC protocol. It is an open architectural model that allows multiple partners like customers, merchants, payment processors, banks, mobile network operators and other technology providers to become part of the payment value-chain and create solutions that complement payments in many unexpected ways.

\section{Factor 7: Mobile payment market cooperation}

Hedman and Henningsson (2015) integrated the market cooperation theory with ecosystems theory from the business technology domain in order to understand the digitalization of payments as an innovation that affects the competition and collaboration among stakeholders in the payment 
ecosystem. According to Hedman and Henningsson (2015), competitors could use mobile payment services either in defensive build-and-defend strategies to protect market position or it could be used in offensive battering-ram strategies for ecosystem entry or position improvement. Liu et al. (2015) examined the changes in technology ecosystem of payments in financial services. They retrospectively analysed the mobile payment services as a new channels for consumer payments for services and goods purchases and other forms of economic exchange in a specific industry. The three building blocks of mobile payment services that provide foundation for digital business includes technology components, technology-based services, and the technology supported infrastructures (Liu et al., 2015). Competition and cooperation impacts the building blocks of mobile payment services and regulatory forces play in driving or delaying innovation. De Reuver et al. (2014) integrated the collective action theory and platform theory to understand the collaboration and competition between banks and telecom operators for trusted service for mobile payment. Issues in collaboration and competition between banks and telecom were due to mobile payment platform characteristics of openness to third party, platform competition and governance of relations with third parties. The major problems for dissolution of the mobile payment services identified were conflicts, differing strategic objectives and interests, governance issues and lack of dependencies.

\section{CONCLUSION}

Assessing the core research themes of mobile payment services research yields intriguing perceptions about the publications during the last two decades. While a number of researchers have studied mobile payment services related literature, a co-citation analysis was yet to be attempted. As many as 406 research articles and 3424 citations were collated from the WoK database. This study revealed that mobile payment services field is an actively changing one, as may be deduced from the manner in which various themes have evolved during the last two decades. From an initial static approach of evaluating mobile payment services baseline and themes, research now aims at the emerging architecture and finally to the adoption and usage through frameworks and models. This indicates a sea of opportunities to enhance our collective knowledge base about mobile payment services, a domain where innovative technologies keep emerging. Hence, to summarise, the present research study investigated the core intellectual structures involved in mobile payment services; as additional articles are published in mobile payment services subfields, this study should serve as a basis for future studies to delve into the evolution of mobile payment services and point out the fundamental themes of sub-topics such as NFC and e-wallet among others.

\section{Limitations and future studies}

Even after employing robust data interpretation methodologies, research attempts cannot be entirely free of limitations. However, these very limitations open avenues for future research and need to be enlisted.

The WoK database is indeed prolific and offers as many as 10000 high-quality journals to the readers. However, journals such as the Journal of Information Technology, and Theory and Application were conspicuous by their absence from this database. Hence, other research studies should particularly target journals missing from the WoK database, and also explore other databases.

In addition, the keywords that were employed in the present study pertained specifically to "mobile payment services". In a dynamic research field such as this, novel terms keep evolving. 
Hence, future researchers should target keywords such as NFC, e-wallet, SIM-based applications, mobile payment and QR Payment (Lai and Chuah, 2010; Leong et al., 2013a). Including such new keywords in the study shall ensure that more relevant mobile payment services-related articles are brought within the scope of the study.

Also, due to page-limit constraints, only the highly cited research articles from the mobile payment services field had to be included in this study.

The publication lag bias mars the otherwise statistically sound co-citation method. It is possible that the study missed out on a few recently published research articles that would emerge as one of the fundamental articles but only in due course of time.

Lastly, all the co-citations could not be displayed, though the highly co-cited ones do indicate the critical publications in the field of mobile payment services.

While conducting this research, the co-citation study was limited to document, author and journal co-citation analysis while concentrating on co-document analysis. While these studies did highlight the core concepts of the mobile payment services research domain, other co-citation studies such as journal co-citation or co-author citation can throw new light on the findings. Mobile payment services have become globally popular. IT/IS adoption, diffusion, acceptance and use have been explained effectively by several theories such as the TRA (Fishbein and Ajzen1975), TPB (Ajzen, 1991), TAM (Davis, 1989) and UTAUT (Venkatesh et al. 2003). Although new mobile payment services have emerged, marketers must understand the diffusion process for their widespread use. Rogers (1995) developed the diffusion of innovations theory to understand the diffusion processes of services or products. Diffusion model has been the focus of only a few studies and hence, may emerge as the target of further studies scrutinizing the global diffusion of mobile payment services.

\section{REFERENCES}

Ajzen, I. (1991). The theory of planned behavior. Organizational behavior and human decision processes, 50(2), $179-211$.

Al-Jabri, I. M., \& Sohail, M. S. (2012). Mobile banking adoption: Application of diffusion of innovation theory.

Apanasevic, T., Markendahl, J., \& Arvidsson, N. (2016). Stakeholders' expectations of mobile payment in retail: lessons from Sweden. International Journal of Bank Marketing, 34(1), 37-61.

Bonacich, P. (1972). Factoring and weighting approaches to status scores and clique identification. Journal of Mathematical Sociology, 2(1), 113-120.

Borgatti, S. P. (2002). NetDraw: Graph visualization software. Harvard: Analytic Technologies.

Cagliano, A. C., Cagliano, A. C., De Marco, A., De Marco, A., Rafele, C., \& Rafele, C. (2017). E-grocery supply chain management enabled by mobile tools. Business Process Management Journal, 23(1), 47-70.

Chen, F., \& Liu, Y. (2016). Research on the risk factors of mobile business: based on the sorting Delphi method. International Journal of Engineering Research in Africa, 21.

Chen, L. D. (2008). A model of consumer acceptance of mobile payment. International Journal of Mobile Communications, 6(1), 32-52.

Cheng, Y. H., \& Huang, T. Y. (2013). High speed rail passengers' mobile ticketing Adoption. Transportation Research Part C: Emerging Technologies, 30, 143-160. 
Constantiou, I. D., Damsgaard, J., \& Knutsen, L. (2006). Exploring perceptions and use of mobile services: user differences in an advancing market. International Journal of Mobile Communications, 4(3), 231-247.

Dahlberg, T., Mallat, N., Ondrus, J., \& Zmijewska, A. (2008). Past, present and future of mobile payments research: A literature review. Electronic Commerce Research and Applications, 7(2), 165-181.

Davis, F. D. (1989). Perceived usefulness, perceived ease of use, and user acceptance of information technology. MIS quarterly, 319-340.

De Kerviler, G., Demoulin, N. T., \& Zidda, P. (2016). Adoption of in-store mobile payment: Are perceived risk and convenience the only drivers?. Journal of Retailing and Consumer Services, 31, 334-344.

de Reuver, M., Verschuur, E., Nikayin, F., Cerpa, N., \& Bouwman, H. (2015). Collective action for mobile payment platforms: A case study on collaboration issues between banks and telecom operators. Electronic Commerce Research and Applications, 14(5), 331-344.

Di Pietro, L., Mugion, R. G., Mattia, G., Renzi, M. F., \& Toni, M. (2015). The integrated model on mobile payment acceptance (IMMPA): an empirical application to public transport. Transportation Research Part C: Emerging Technologies, 56, 463-479.

Donner, J., \& Tellez, C. A. (2008). Mobile banking and economic development: Linking adoption, impact, and use. Asian journal of communication, 18(4), 318-332.

Duane, A., O’Reilly, P., \& Andreev, P. (2014). Realising M-Payments: modelling consumers' willingness to M-pay using Smart Phones. Behaviour \& Information Technology, 33(4), 318-334.

Falahati, A., \& Jannati, H. (2015). All-or-nothing approach to protect a distance bounding protocol against terrorist fraud attack for low-cost devices. Electronic Commerce Research, 15(1), 75-95.

Fernandes, C., Ferreira, J. J., Raposo, M. L., Estevão, C., Peris-Ortiz, M., \& Rueda-Armengot, C. (2017). The dynamic capabilities perspective of strategic management: a co-citation analysis. Scientometrics, 1-27.

Ferreira, J. J. M., Fernandes, C. I., \& Ratten, V. (2016). A co-citation bibliometric analysis of strategic management research. Scientometrics, 109(1), 1-32.

Fishbein, M., \& Ajzen, I. (1977). Belief, attitude, intention, and behavior: An introduction to theory and research.

Francisco, L. C., Francisco, M. L., \& Juan, S. F. (2015). Payment systems in new electronic environments: consumer behavior in payment systems via SMS. International Journal of Information Technology \& Decision Making, 14(02), 421-449.

Funk, J. L. (2007). Solving the startup problem in Western mobile Internet markets. Telecommunications Policy, 31(1), 14-30.

Gao, L., \& Waechter, K. A. (2017). Examining the role of initial trust in user adoption of mobile payment services: an empirical investigation. Information Systems Frontiers, 19(3), 525-548.

Gerpott, T. J., \& Meinert, P. (2016). Correlates of using the billing system of a mobile network operator to pay for digital goods and services. Information Systems Frontiers, 18(6), 1265-1283.

Hair, J. F., Black, W. C., Babin, B. J., Anderson, R. E., \& Tatham, R. L. (1998). Multivariate data analysis (Vol. 5, No. 3, pp. 207-219). Upper Saddle River, NJ: Prentice hall.

Han, J., Yang, Y., Huang, X., Yuen, T. H., Li, J., \& Cao, J. (2016). Accountable mobile E-commerce scheme via identity-based plaintext-checkable encryption. Information Sciences, 345, 143-155.

Hedman, J., \& Henningsson, S. (2015). The new normal: Market cooperation in the mobile payments ecosystem. Electronic Commerce Research and Applications, 14(5), 305-318. 
Hillman, S., \& Neustaedter, C. (2017). Trust and mobile commerce in North America. Computers in Human Behavior, 70, 10-21.

Hsiao, C. H., \& Yang, C. (2011). The intellectual development of the technology acceptance model: A cocitation analysis. International Journal of Information Management, 31(2), 128-136.

Huang, X., Dai, X., \& Liang, W. (2014). BulaPay: a novel web service based third-party payment system for e-commerce. Electronic Commerce Research, 14(4), 611-633.

Hwang, R. J., Shiau, S. H., \& Jan, D. F. (2007). A new mobile payment scheme for roaming services. Electronic Commerce Research and Applications, 6(2), 184-191.

Isaac, J. T., \& Zeadally, S. (2014). Design, implementation, and performance analysis of a secure payment protocol in a payment gateway centric model. Computing, 96(7), 587-611.

Javan, S. L., \& Bafghi, A. G. (2014). An anonymous mobile payment protocol based on SWPP. Electronic Commerce Research, 14(4), 635-660.

Kalinic, Z., \& Marinkovic, V. (2016). Determinants of users' intention to adopt m-commerce: an empirical analysis. Information Systems and e-Business Management, 14(2), 367-387.

Kapoor, K. K., Dwivedi, Y. K., \& Williams, M. D. (2015). Examining the role of three sets of innovation attributes for determining adoption of the interbank mobile payment service. Information Systems Frontiers, 17(5), 1039-1056.

Karjaluoto, H., Töllinen, A., Pirttiniemi, J., \& Jayawardhena, C. (2014). Intention to use mobile customer relationship management systems. Industrial Management \& Data Systems, 114(6), 966-978.

Karnouskos, S. (2004). Mobile payment: a journey through existing procedures and standardization initiatives. IEEE Communications Surveys \& Tutorials, 6(4).

Keramati, A., Taeb, R., Larijani, A. M., \& Mojir, N. (2012). A combinative model of behavioural and technical factors affecting 'Mobile'-payment services adoption: an empirical study. The Service Industries Journal, 32(9), 1489-1504.

Khalilzadeh, J., Ozturk, A. B., \& Bilgihan, A. (2017). Security-related factors in extended UTAUT model for NFC based mobile payment in the restaurant industry. Computers in Human Behavior, 70, 460-474.

Khan, G. F., \& Wood, J. (2015). Information technology management domain: Emerging themes and keyword analysis. Scientometrics, 105(2), 959-972.

Kim, C., Mirusmonov, M., \& Lee, I. (2010). An empirical examination of factors influencing the intention to use mobile payment. Computers in Human Behavior, 26(3), 310-322.

Kim, C., Tao, W., Shin, N., \& Kim, K. S. (2010). An empirical study of customers' perceptions of security and trust in e-payment systems. Electronic commerce research and applications, 9(1), 84-95.

Koenig-Lewis, N., Marquet, M., Palmer, A., \& Zhao, A. L. (2015). Enjoyment and social influence: predicting mobile payment adoption. The Service Industries Journal, 35(10), 537-554.

Kujala, S., Mugge, R., \& Miron-Shatz, T. (2017). The role of expectations in service evaluation: A longitudinal study of a proximity mobile payment service. International Journal of Human-Computer Studies, 98, 5161.

Lee, J. S., Chang, Y. F., \& Chang, C. C. (2008). Secure authentication protocols for mobile commerce transactions. International Journal of Innovative Computing, Information and Control, 4(9), 2305-2314.

Li, W., Wen, Q., Su, Q., \& Jin, Z. (2012). An efficient and secure mobile payment protocol for restricted connectivity scenarios in vehicular ad hoc network. Computer Communications, 35(2), 188-195. 
Liang, T. P., \& Yeh, Y. H. (2011). Effect of use contexts on the continuous use of mobile services: the case of mobile games. Personal and Ubiquitous Computing, 15(2), 187-196.

Liébana-Cabanillas, F., Muñoz-Leiva, F., \& Sánchez-Fernández, J. (2015). Behavioral Model of Younger Users in M-Payment Systems. Journal of Organizational Computing and Electronic Commerce, 25(2), 169-190.

Liébana-Cabanillas, F., Ramos de Luna, I., \& Montoro-Ríos, F. (2017). Intention to use new mobile payment systems: a comparative analysis of SMS and NFC payments. Economic Research-Ekonomska Istraživanja, 30(1), 892-910.

Liébana-Cabanillas, F., Ramos de Luna, I., \& Montoro-Ríos, F. J. (2015) (b). User behaviour in QR mobile payment system: the QR Payment Acceptance Model. Technology Analysis \& Strategic Management, 27(9), 1031-1049.

Liébana-Cabanillas, F., Sánchez-Fernández, J., \& Muñoz-Leiva, F. (2014). Antecedents of the adoption of the new mobile payment systems: The moderating effect of age. Computers in Human Behavior, 35, 464-478.

Liébana-Cabanillas, F., Sánchez-Fernández, J., \& Muñoz-Leiva, F. (2014) (b). The moderating effect of experience in the adoption of mobile payment tools in Virtual Social Networks: The m-Payment Acceptance Model in Virtual Social Networks (MPAM-VSN). International Journal of Information Management, 34(2), 151-166.

Liu, D., Tong, C., Liu, Y., Yuan, Y., \& Ju, C. (2016). Examining the adoption and continuous usage of contextaware services: An empirical study on the use of an intelligent tourist guide. Information Development, 32(3), 608-621.

Liu, J., Kauffman, R. J., \& Ma, D. (2015). Competition, cooperation, and regulation: Understanding the evolution of the mobile payments technology ecosystem. Electronic Commerce Research and Applications, 14(5), 372-391.

Liu, Y., \& Li, H. (2011). Exploring the impact of use context on mobile hedonic services adoption: An empirical study on mobile gaming in China. Computers in Human Behavior, 27(2), 890-898.

Lu, J., Wei, J., Yu, C. S., \& Liu, C. (2017). How do post-usage factors and espoused cultural values impact mobile payment continuation?. Behaviour \& Information Technology, 36(2), 140-164.

Luna, I. R. D., Montoro-Ríos, F., Liébana-Cabanillas, F., \& Luna, J. G. D. (2017). NFC technology acceptance for mobile payments: A Brazilian Perspective. Revista brasileira de gestão de negócios, 19(63), 82-103.

Madan, K., Madan, K., Yadav, R., \& Yadav, R. (2016). Behavioural intention to adopt mobile wallet: a developing country perspective. Journal of Indian Business Research, 8(3), 227-244.

Madureira, A. (2017). Factors that hinder the success of SIM-based mobile NFC service deployments. Telematics and Informatics, 34(1), 133-150.

Mallat, N., \& Tuunainen, V. K. (2008). Exploring merchant adoption of mobile payment systems: An empirical study. E-service Journal, 6(2), 24-57.

Mallat, N., Rossi, M., Tuunainen, V. K., \& Öörni, A. (2009). The impact of use context on mobile services acceptance: The case of mobile ticketing. Information \& management, 46(3), 190-195.

Martínez-Peláez, R., Toral-Cruz, H., Ruiz, J., \& Velarde-Alvarado, P. (2015). P2PM-pay: Person to person mobile payment scheme controlled by expiration date. Wireless Personal Communications, 85(1), 289-304.

McCain, K. W. (1990). Mapping authors in intellectual space: A technical overview. Journal of the American society for information science, $41(6), 433$.

Miao, M., \& Jayakar, K. (2016). Mobile payments in Japan, South Korea and China: Cross-border convergence or divergence of business models?. Telecommunications Policy, 40(2), 182-196. 
Molina-Castillo, F. J., Rodriguez-Guirao, A., Lopez-Nicolas, C., \& Bouwman, H. (2016). Analysis of mobile pre-payment (pay in advance) and post-payment (pay later) services. International Journal of Mobile Communications, 14(5), 499-517.

Moorthy, K., Ling, C. S., Fatt, Y. W., Yee, C. M., Yin, E. C. K., Yee, K. S., \& Wei, L. K. (2017). Barriers of Mobile Commerce Adoption Intention: Perceptions of Generation X in Malaysia. Journal of Theoretical and Applied Electronic Commerce Research, 12(2), 37-53.

Musa, A., Khan, H. U., \& AlShare, K. A. (2015). Factors influence consumers' adoption of mobile payment devices in Qatar. International journal of mobile communications, 13(6), 670-689.

Ngai, E. W., \& Gunasekaran, A. (2007). A review for mobile commerce research and applications. Decision support systems, 43(1), 3-15.

O'Reilly, P., Duane, A., \& Andreev, P. (2012). To M-Pay or not to M-Pay—Realising the potential of smart phones: conceptual modeling and empirical validation. Electronic Markets, 22(4), 229-241.

Oliveira, T., Thomas, M., Baptista, G., \& Campos, F. (2016). Mobile payment: Understanding the determinants of customer adoption and intention to recommend the technology. Computers in Human Behavior, 61, 404414.

Ooi, K. B., \& Tan, G. W. H. (2016). Mobile technology acceptance model: An investigation using mobile users to explore smartphone credit card. Expert Systems with Applications, 59, 33-46.

Pham, T. T. T., \& Ho, J. C. (2015). The effects of product-related, personal-related factors and attractiveness of alternatives on consumer adoption of NFC-based mobile payments. Technology in Society, 43, 159-172.

Phonthanukitithaworn, C., Sellitto, C., \& Fong, M. W. (2016). A Comparative Study of Current and Potential Users of Mobile Payment Services. SAGE Open, 6(4). DOI: 2158244016675397.

Phonthanukitithaworn, C., Sellitto, C., \& Fong, M. W. (2016) (b). An investigation of mobile payment (m-payment) services in Thailand. Asia-Pacific Journal of Business Administration, 8(1), 37-54.

Pilkington, A., \& Meredith, J. (2009). The evolution of the intellectual structure of operations management-1980-2006: A citation/co-citation analysis. Journal of Operations Management, 27(3), 185202.

Pousttchi, K., Schiessler, M., \& Wiedemann, D. G. (2009). Proposing a comprehensive framework for analysis and engineering of mobile payment business models. Information Systems and E-Business Management, 7(3), 363-393.

Ramos-de-Luna, I., Montoro-Ríos, F., \& Liébana-Cabanillas, F. (2016). Determinants of the intention to use NFC technology as a payment system: an acceptance model approach. Information Systems and e-Business Management, 14(2), 293-314.

Ramos-Rodríguez, A. R., \& Ruíz-Navarro, J. (2004). Changes in the intellectual structure of strategic management research: A bibliometric study of the Strategic Management Journal, 1980-2000. Strategic Management Journal, 25(10), 981-1004.

Rodrigues, H., José, R., Coelho, A., Melro, A., Ferreira, M. C., Monteiro, M. P., \& Ribeiro, C. (2014). MobiPag: Integrated Mobile Payment, Ticketing and Couponing Solution Based on NFC. Sensors, 14(8), 1338913415.

Rogers, E. M. (2003). Diffusion of innovations. Free Press. New York, 551.

Rouibah, K., Lowry, P. B., \& Hwang, Y. (2016). The effects of perceived enjoyment and perceived risks on trust formation and intentions to use online payment systems: New perspectives from an Arab country. Electronic Commerce Research and Applications, 19, 33-43. 
Ruiz-del-Olmo, F. J. (2014). Young People as Users of Branded Applications on Mobile Devices/Los jóvenes como usuarios de aplicaciones de marca en dispositivos móviles. Comunicar (English edition), 22(43), 73-81.

Schierz, P. G., Schilke, O., \& Wirtz, B. W. (2010). Understanding consumer acceptance of mobile payment services: An empirical analysis. Electronic commerce research and applications, 9(3), 209-216.

Seol, S., Lee, H., \& Zo, H. (2016). Exploring factors affecting the adoption of mobile office in business: an integration of TPB with perceived value. International Journal of Mobile Communications, 14(1), 1-25.

Shaikh, A. A., \& Karjaluoto, H. (2015). Mobile banking adoption: A literature review. Telematics and Informatics, 32(1), 129-142.

Shi, F., Liu, W., Jin, D., \& Song, J. (2013). A countermeasure against wormhole attacks in MANETs using analytical hierarchy process methodology. Electronic Commerce Research, 13(3), 329-345.

Shiau, W. L. (2016). The intellectual core of enterprise information systems: a co-citation analysis. Enterprise Information Systems, 10(8), 815-844.

Shiau, W. L., \& Dwivedi, Y. K. (2013). Citation and co-citation analysis to identify core and emerging knowledge in electronic commerce research. Scientometrics, 94(3), 1317-1337.

Shin, D. H. (2010). Modeling the interaction of users and mobile payment system: Conceptual framework. International Journal of Human-Computer Interaction, 26(10), 917-940.

Silic, M., \& Back, A. (2016). What are the Keys to a Successful Mobile Payment System? Case of Cytizi: Mobile Payment System. Journal of Global Information Management (JGIM), 24(3), 1-20.

Slade, E. L., Dwivedi, Y. K., Piercy, N. C., \& Williams, M. D. (2015). Modeling consumers' adoption intentions of remote mobile payments in the United Kingdom: extending UTAUT with innovativeness, risk, and trust. Psychology \& Marketing, 32(8), 860-873.

Small, H. (1973). Co-citation in the scientific literature: A new measure of the relationship between two documents. Journal of the Association for Information Science and Technology, 24(4), 265-269.

Sripalawat, J., Thongmak, M., \& Ngramyarn, A. (2011). M-banking in metropolitan Bangkok and a comparison with other countries. Journal of computer information systems, 51(3), 67-76.

Tabachnick, B. G., Fidell, L. S., \& Osterlind, S. J. (2001). Using multivariate statistics.

Teo, A. C., Tan, G. W. H., Ooi, K. B., \& Lin, B. (2015). Why consumers adopt mobile payment? A partial least squares structural equation modelling (PLS-SEM) approach. International Journal of Mobile Communications, 13(5), 478-497.

Teo, A. C., Tan, G. W. H., Ooi, K. B., Hew, T. S., \& Yew, K. T. (2015) (b). The effects of convenience and speed in m-payment. Industrial Management \& Data Systems, 115(2), 311-331.

Thakur, R., \& Srivastava, M. (2014). Adoption readiness, personal innovativeness, perceived risk and usage intention across customer groups for mobile payment services in India. Internet Research, 24(3), 369-392.

Upadhyay, P., \& Chattopadhyay, M. (2015). Examining mobile based payment services adoption issues: A new approach using hierarchical clustering and self-organizing maps. Journal of Enterprise Information Management, 28(4), 490-507.

Upadhyay, P., \& Jahanyan, S. (2016). Analyzing user perspective on the factors affecting use intention of mobile based transfer payment. Internet Research, 26(1), 38-56.

Venkatesh, V., Morris, M. G., Davis, G. B., \& Davis, F. D. (2003). User acceptance of information technology: Toward a unified view. MIS quarterly, 425-478. 
Wan, J., Zou, C., Zhou, K., Lu, R., \& Li, D. (2014). IoT sensing framework with inter-cloud computing capability in vehicular networking. Electronic Commerce Research, 14(3), 389-416.

Wang, C. H., \& Chen, S. C. (2013). Bibliometric and Social Network Analysis for Data Mining: The Intellectual Structure of Tourism Destination Literature. Journal of Testing and Evaluation, 42(1), 229-241.

Webster, J., \& Watson, R. T. (2002). Analyzing the past to prepare for the future: Writing a literature review. MIS quarterly, xiii-xxiii.

White, H. D., \& Griffith, B. C. (1981). Author cocitation: A literature measure of intellectual structure. Journal of the Association for Information Science and Technology, 32(3), 163-171.

Xin, H., Techatassanasoontorn, A. A., \& Tan, F. B. (2015). Antecedents of consumer trust in mobile payment adoption. Journal of Computer Information Systems, 55(4), 1-10.

Yang, J. H., \& Chang, C. C. (2012). A low computational-cost electronic payment scheme for mobile commerce with large-scale mobile users. Wireless Personal Communications, 63(1), 83-99.

Yang, J. H., Chang, Y. F., \& Chen, Y. H. (2013). An efficient authenticated encryption scheme based on ECC and its application for electronic payment. Information Technology and Control, 42(4), 315-324.

Yang, L., \& Lou, W. (2016). A contract-ruled economic model for QoS guarantee in mobile peer-to-peer streaming services. IEEE Transactions on Mobile Computing, 15(5), 1047-1061.

Yang, M. (2013). Churn management and policy: Measuring the effectiveness of fixed-mobile bundling on mobile subscriber retention. Journal of Media Economics, 26(4), 170-185.

Yang, M., Li, Y., Jin, D., Yuan, J., You, I., \& Zeng, L. (2014). Opportunistic sharing scheme for spectrum allocation in wireless virtualization. Soft Computing, 18(9), 1685-1696.

Yang, S. (2016). Role of transfer-based and performance-based cues on initial trust in mobile shopping services: a cross-environment perspective. Information Systems and e-Business Management, 14(1), 47-70.

Yang, S., Lu, Y., Chau, P. Y., \& Gupta, S. (2017). Role of channel integration on the service quality, satisfaction, and repurchase intention in a multi-channel (online-cum-mobile) retail environment. International Journal of Mobile Communications, 15(1), 1-25.

Yang, S., Lu, Y., Gupta, S., Cao, Y., \& Zhang, R. (2012). Mobile payment services adoption across time: An empirical study of the effects of behavioral beliefs, social influences, and personal traits. Computers in Human Behavior, 28(1), 129-142.

Yang, S., Wang, Y., \& Wei, J. (2014) (b). Integration and consistency between web and mobile services. Industrial Management \& Data Systems, 114(8), 1246-1269.

Yang, Y., Liu, Y., Li, H., \& Yu, B. (2015). Understanding perceived risks in mobile payment acceptance. Industrial Management \& Data Systems, 115(2), 253-269.

Zhong, S., \& Wu, F. (2010). A collusion-resistant routing scheme for noncooperative wireless ad hoc networks. IEEE/ACM Transactions on Networking, 18(2), 582-595.

Zhou, T. (2013). An empirical examination of continuance intention of mobile payment services. Decision Support Systems, 54(2), 1085-1091.

Zhou, T. (2014). An empirical examination of initial trust in mobile payment. Wireless personal communications, 77(2), 1519-1531.

Zhou, T., Lu, Y., \& Wang, B. (2010). Integrating TTF and UTAUT to explain mobile banking user adoption. Computers in human behavior, 26(4), 760-767. 
Appendix: Cluster 1: Mobile payment services adoption and usage

\begin{tabular}{|c|c|c|c|c|c|c|c|c|c|c|c|c|c|c|c|c|c|c|c|c|c|c|c|c|c|c|c|c|}
\hline \multirow[t]{2}{*}{ Authors } & \multirow{2}{*}{$\begin{array}{l}\text { Theories/ } \\
\text { Models }\end{array}$} & \multirow{2}{*}{$\begin{array}{l}\text { Dependent } \\
\text { variables }\end{array}$} & \multicolumn{26}{|c|}{ Independent variables } \\
\hline & & & 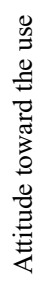 & 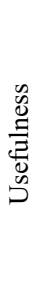 & 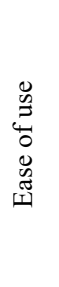 & 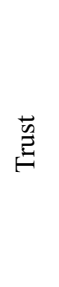 & $\frac{u}{a}$ & 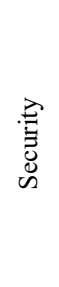 & 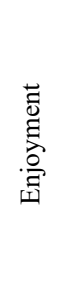 & 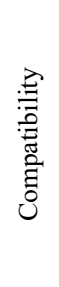 & 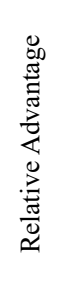 & 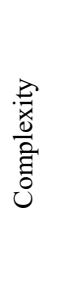 & 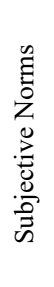 & 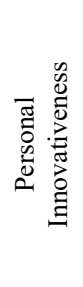 & 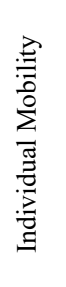 & 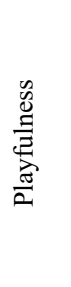 & 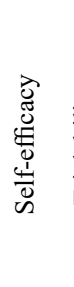 & 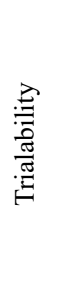 & 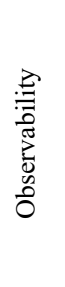 & 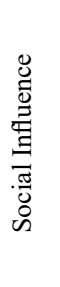 & 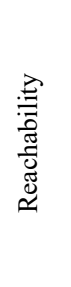 & 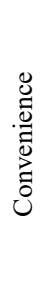 & 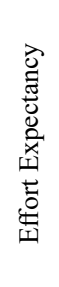 & 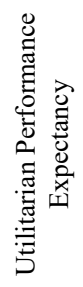 & 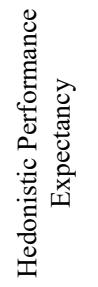 & 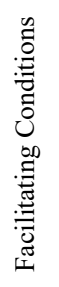 & 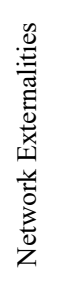 & 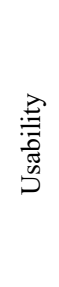 \\
\hline De Luna et al. (2016) & TAM & $\begin{array}{l}\text { Intention to use } \\
\text { NFC payments } \\
\text { systems }\end{array}$ & $\checkmark$ & $\checkmark$ & $\checkmark$ & & & $\checkmark$ & & $\checkmark$ & & & $\checkmark$ & $\checkmark$ & $\checkmark$ & & & & & & & & & & & & & \\
\hline Schierz et al. (2010) & TAM & Intention to use & $\checkmark$ & $\checkmark$ & $\checkmark$ & & & $\checkmark$ & & $\checkmark$ & & & $\checkmark$ & & $\checkmark$ & & & & & & & & & & & & & \\
\hline Zhou (2013) & $\begin{array}{c}\text { Information } \\
\text { systems success } \\
\text { model }\end{array}$ & $\begin{array}{l}\text { Continuance } \\
\text { intention }\end{array}$ & & & & $\checkmark$ & & & & & & & & & & & & & & & & & & & & & & \\
\hline Seol et al. (2016) & $\begin{array}{c}\text { TPB, } \\
\text { Perceived Value }\end{array}$ & $\begin{array}{l}\text { Intention } \\
\text { to use }\end{array}$ & $\checkmark$ & $\checkmark$ & & & $\checkmark$ & & & & & & $\checkmark$ & & & & $\checkmark$ & & & & & & & & & & & \\
\hline Shin (2010) & TAM, UTAUT & Use Behaviour & $\checkmark$ & & & $\checkmark$ & $\checkmark$ & & & & & & $\checkmark$ & & & & & & & & & $\checkmark$ & & & & & & \\
\hline Slade et al. (2015) & UTAUT & $\begin{array}{l}\text { Behavioural } \\
\text { Intention }\end{array}$ & & & & $\checkmark$ & $\checkmark$ & & & & & & & $\checkmark$ & & & & & & $\checkmark$ & & & $\checkmark$ & & & & & \\
\hline Sripalawat et al. (2011) & TAM, ТРВ & Actual use & & $\checkmark$ & $\checkmark$ & & $\checkmark$ & & & & & & $\checkmark$ & & & & $\checkmark$ & & & & & & & & & & & \\
\hline Teo et al. (2015) & UTAUT & $\begin{array}{l}\text { Behavioural } \\
\text { Intention }\end{array}$ & & & & & & & & & & & & $\checkmark$ & & & & & & $\checkmark$ & & & $\checkmark$ & & & $\checkmark$ & & \\
\hline Teo et al. (2015) (b) & UTAUT & $\begin{array}{l}\text { Behavioural } \\
\text { Intention }\end{array}$ & & & & $\checkmark$ & & & & & & & & & & & & & & $\checkmark$ & & & $\checkmark$ & & & $\checkmark$ & & \\
\hline $\begin{array}{l}\text { Upadhyay and } \\
\text { Chattopadhyay (2015) }\end{array}$ & TAM, TTF & Usage intention & & $\checkmark$ & $\checkmark$ & & & & & & & & & $\checkmark$ & & & & & & & & & & & & & & \\
\hline $\begin{array}{l}\text { Upadhyay and } \\
\text { Jahanyan (2016) }\end{array}$ & TAM, TTF & Intention to use & & $\checkmark$ & $\checkmark$ & & & & & & & & & $\checkmark$ & & & & & & & & & & & & & & \\
\hline
\end{tabular}


Appendix: Cont

\begin{tabular}{|c|c|c|c|c|c|c|c|c|c|c|c|c|c|c|c|c|c|c|c|c|c|c|c|c|c|c|c|c|}
\hline \multirow[t]{2}{*}{ Authors } & \multirow{2}{*}{$\begin{array}{l}\text { Theories/ } \\
\text { Models }\end{array}$} & \multirow{2}{*}{$\begin{array}{l}\text { Dependent } \\
\text { variables }\end{array}$} & \multicolumn{26}{|c|}{ Independent variables } \\
\hline & & & 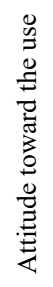 & 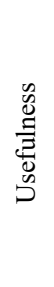 & 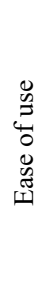 & 蒙 & $\frac{4}{a}$ & 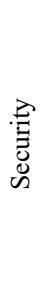 & 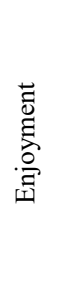 & 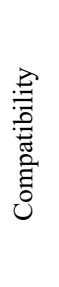 & 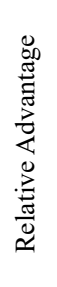 & 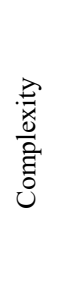 & 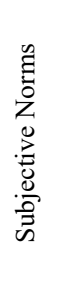 & 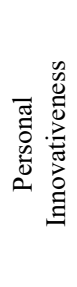 & 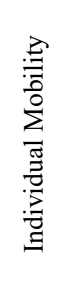 & 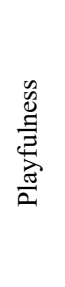 & 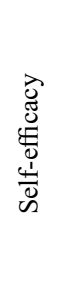 & 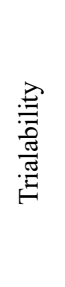 & $\begin{array}{l}\stackrel{3}{\Xi} \\
\stackrel{0}{0} \\
\stackrel{0}{0} \\
0 \\
0\end{array}$ & 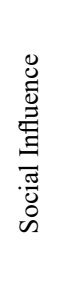 & 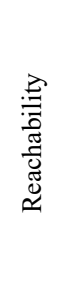 & 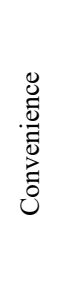 & 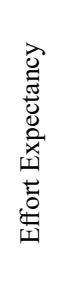 & 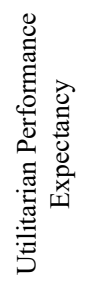 & 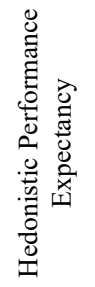 & 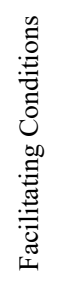 & 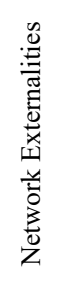 & 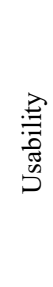 \\
\hline Yang et al. (2012) & TAM, DOI, TPB & $\begin{array}{l}\text { Behavioural } \\
\text { Intention }\end{array}$ & & & & & $\checkmark$ & & & $\checkmark$ & $\checkmark$ & & $\checkmark$ & & & & & & & & & & & & & & & \\
\hline Yang et al. (2014) & $\begin{array}{l}\text { Categorization } \\
\text { theory, } \\
\text { Motivation } \\
\text { theory }\end{array}$ & & & & & & & & & & & & & & & & & & & & & & & & & & & \\
\hline Zhou et al. (2010) & TTF, UTAUT & Adoption & & & & & & & & & & & & & & & & & & $\checkmark$ & & & $\checkmark$ & & $\checkmark$ & $\checkmark$ & & \\
\hline $\begin{array}{l}\text { Madan and Yadav } \\
\text { (2016) }\end{array}$ & UTAUT & $\begin{array}{l}\text { Behavioural } \\
\text { Intention }\end{array}$ & & & & $\checkmark$ & $\checkmark$ & & & & & & & & & & & & & $\checkmark$ & & & $\checkmark$ & & & $\checkmark$ & & \\
\hline $\begin{array}{l}\text { Molina-Castillo et al. } \\
\text { (2016) }\end{array}$ & $\begin{array}{c}\text { TAM, } \\
\text { Motivation } \\
\text { Theory, Self- } \\
\text { efficacy Theory }\end{array}$ & $\begin{array}{l}\text { Behavioural } \\
\text { Intention }\end{array}$ & & $\checkmark$ & $\checkmark$ & & & & & & & & & & & & $\checkmark$ & & & & & & & & & & & \\
\hline Musa et al. (2015) & UTAUT & $\begin{array}{l}\text { Behavioural } \\
\text { Intention }\end{array}$ & & & & & & $\checkmark$ & & & & & $\checkmark$ & & & & & & & & & & & & & & & \\
\hline Oliveira et al. (2016) & UTAUT2, DOI & $\begin{array}{l}\text { Behavioural } \\
\text { Intention }\end{array}$ & & & & & & $\checkmark$ & & $\checkmark$ & & & & $\checkmark$ & & & & & & $\checkmark$ & & & & & $\checkmark$ & $\checkmark$ & & \\
\hline Ooi and Tan (2016) & TAM, DOI & $\begin{array}{l}\text { Behavioural } \\
\text { Intention }\end{array}$ & & $\checkmark$ & $\checkmark$ & $\checkmark$ & $\checkmark$ & & & $\checkmark$ & & & & & & & & & & & & & & & & & & \\
\hline $\begin{array}{l}\text { Phonthanukitithaworn } \\
\text { et al. (2016) }\end{array}$ & TAM, DOI & $\begin{array}{l}\text { Behavioural } \\
\text { Intention }\end{array}$ & & $\checkmark$ & $\checkmark$ & $\checkmark$ & $\checkmark$ & & & $\checkmark$ & & & $\checkmark$ & & & & & & & & & & & & & & & \\
\hline $\begin{array}{l}\text { Phonthanukitithaworn } \\
\text { et al. (2016) (b) }\end{array}$ & TAM, TPB, DOI & $\begin{array}{l}\text { Behavioural } \\
\text { Intention }\end{array}$ & & $\checkmark$ & $\checkmark$ & $\checkmark$ & $\checkmark$ & & & $\checkmark$ & & & $\checkmark$ & & & & & & & & & & & & & & & \\
\hline Lu et al. (2016) & UTAUT & $\begin{array}{l}\text { Continuance } \\
\text { intention }\end{array}$ & & $\checkmark$ & & & & & & & & & & & $\checkmark$ & & & & & $\checkmark$ & & & & & & & & \\
\hline
\end{tabular}




\section{Appendix: Cont.}

\begin{tabular}{|c|c|c|c|c|c|c|c|c|c|c|c|c|c|c|c|c|c|c|c|c|c|c|c|c|c|c|c|c|}
\hline \multirow[t]{2}{*}{ Authors } & \multirow{2}{*}{$\begin{array}{l}\text { Theories/ } \\
\text { Models }\end{array}$} & \multirow{2}{*}{$\begin{array}{l}\text { Dependent } \\
\text { variables }\end{array}$} & \multicolumn{26}{|c|}{ Independent variables } \\
\hline & & & 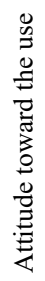 & 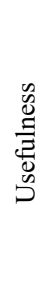 & 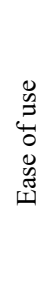 & $\stackrel{\overrightarrow{0}}{E}$ & $\frac{y}{a}$ & 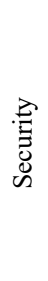 & 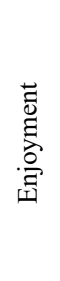 & 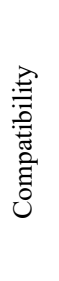 & 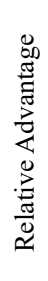 & 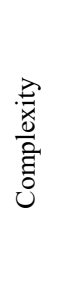 & 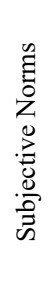 & 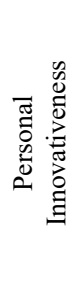 & 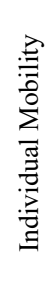 & 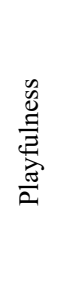 & 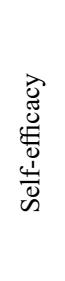 & 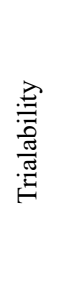 & 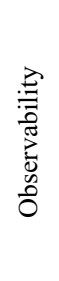 & 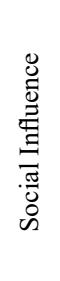 & 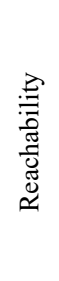 & 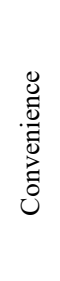 & 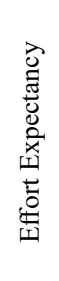 & 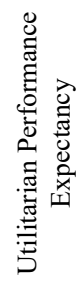 & 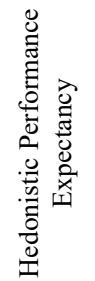 & 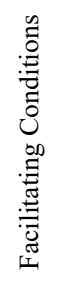 & 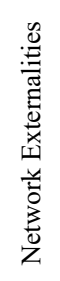 & 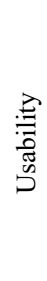 \\
\hline Liang and Yeh (2010) & TAM, TRA & $\begin{array}{l}\text { Continuance } \\
\text { intention }\end{array}$ & $\checkmark$ & & $\checkmark$ & & & & & & & & $\checkmark$ & & & $\checkmark$ & & & & & & & & & & & & \\
\hline $\begin{array}{l}\text { Liébana-Cabanillas } \\
\text { et al. (2014) }\end{array}$ & TAM, TRA & $\begin{array}{l}\text { Intention } \\
\text { to use }\end{array}$ & $\checkmark$ & $\checkmark$ & $\checkmark$ & $\checkmark$ & $\checkmark$ & & & & & & $\checkmark$ & & & & & & & $\checkmark$ & & & & & & & & \\
\hline $\begin{array}{l}\text { Liébana-Cabanillas } \\
\text { et al. (2014) (b) }\end{array}$ & TAM, TRA & $\begin{array}{l}\text { Intention } \\
\text { to use }\end{array}$ & $\checkmark$ & $\checkmark$ & $\checkmark$ & $\checkmark$ & $\checkmark$ & & & & & & $\checkmark$ & & & & & & & $\checkmark$ & & & & & & & & \\
\hline $\begin{array}{l}\text { Liébana-Cabanillas } \\
\text { et al. (2015) }\end{array}$ & TAM & $\begin{array}{l}\text { Intention } \\
\text { to use the } \\
\text { QR system }\end{array}$ & $\checkmark$ & $\checkmark$ & $\checkmark$ & & & $\checkmark$ & & $\checkmark$ & & & $\checkmark$ & $\checkmark$ & $\checkmark$ & & & & & & & & & & & & & \\
\hline $\begin{array}{l}\text { Liébana-Cabanillas } \\
\text { et al. (2015) (b) }\end{array}$ & TAM & $\begin{array}{l}\text { Intention } \\
\text { to use }\end{array}$ & & $\checkmark$ & $\checkmark$ & $\checkmark$ & $\checkmark$ & & & & & & & & & & & & & & & & & & & & & \\
\hline $\begin{array}{l}\text { Liébana-Cabanillas } \\
\text { et al. (2017) }\end{array}$ & TAM, TRA & $\begin{array}{l}\text { Intention } \\
\text { to use }\end{array}$ & $\checkmark$ & $\checkmark$ & $\checkmark$ & & & $\checkmark$ & & & & & $\checkmark$ & & & & & & & & & & & & & & & \\
\hline Liu and Li (2011) & TAM & $\begin{array}{l}\text { Behavioural } \\
\text { Intention }\end{array}$ & & $\checkmark$ & $\checkmark$ & & & & $\checkmark$ & & & & & & & & & & & & & & & & & & & \\
\hline Liu et al. (2016) & TAM & $\begin{array}{l}\text { Continue } \\
\text { usage }\end{array}$ & & & & & & & $\checkmark$ & & $\checkmark$ & & & & & & & & & & & & & & & & & \\
\hline Kujala et al. (2017) & TAM & $\begin{array}{l}\text { Behavioural } \\
\text { Intention }\end{array}$ & & & & & & & $\checkmark$ & & & & & & & & & & & & & & & & & & & $\checkmark$ \\
\hline $\begin{array}{l}\text { Kalinic and Marinkovic } \\
\text { (2015) }\end{array}$ & TAM & $\begin{array}{l}\text { Behavioural } \\
\text { Intention }\end{array}$ & & $\checkmark$ & $\checkmark$ & & & & & & & & & $\checkmark$ & $\checkmark$ & & & & & $\checkmark$ & & & & & & & & \\
\hline $\begin{array}{l}\text { Liébana-Cabanillas } \\
\text { et al. (2014) }\end{array}$ & TAM, TRA & Intention to use & $\checkmark$ & $\checkmark$ & $\checkmark$ & $\checkmark$ & $\checkmark$ & & & & & & $\checkmark$ & & & & & & & & & & & & & & & \\
\hline
\end{tabular}


Appendix: Cont.

\begin{tabular}{|c|c|c|c|c|c|c|c|c|c|c|c|c|c|c|c|c|c|c|c|c|c|c|c|c|c|c|c|c|}
\hline \multirow[t]{2}{*}{ Authors } & \multirow[t]{2}{*}{$\begin{array}{l}\text { Theories/ } \\
\text { Models }\end{array}$} & \multirow[t]{2}{*}{$\begin{array}{l}\text { Dependent } \\
\text { variables }\end{array}$} & \multicolumn{26}{|c|}{ Independent variables } \\
\hline & & & 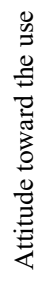 & 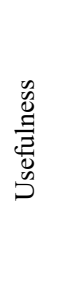 & 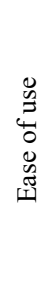 & $\vec{E}$ & $\frac{\pi}{\tilde{a}}$ & 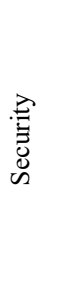 & 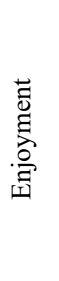 & 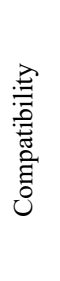 & 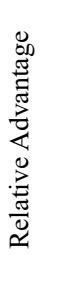 & 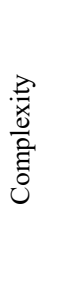 & 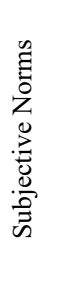 & 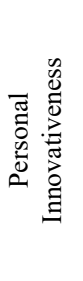 & 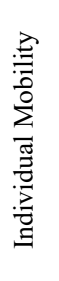 & 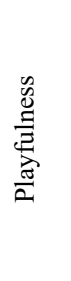 & 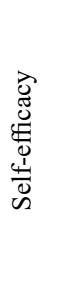 & 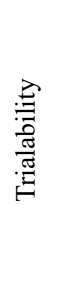 & 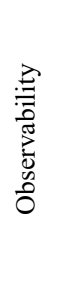 & 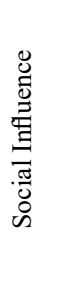 & 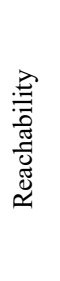 & 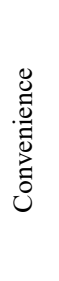 & 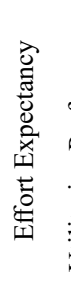 & 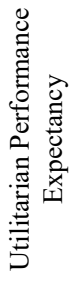 & 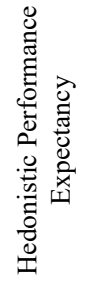 & 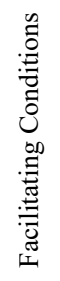 & 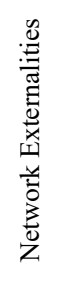 & 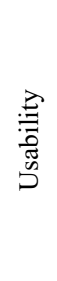 \\
\hline Kapoor et al. (2014) & DOI & $\begin{array}{l}\text { Behavioural } \\
\text { Intention }\end{array}$ & & & & & $\checkmark$ & & & $\checkmark$ & $\checkmark$ & $\checkmark$ & & & & & & $\checkmark$ & $\checkmark$ & & & & & & & & & \\
\hline Karjaluoto et al. (2014) & TAM & $\begin{array}{l}\text { Behavioural } \\
\text { Intention }\end{array}$ & $\checkmark$ & $\checkmark$ & $\checkmark$ & & $\checkmark$ & & & & & & & $\checkmark$ & & & & & & & $\checkmark$ & & & & & & & \\
\hline Keramati et al. (2012) & DOI, TPB & $\begin{array}{l}\text { Behavioural } \\
\& \text { Technical }\end{array}$ & & $\checkmark$ & $\checkmark$ & $\checkmark$ & & $\checkmark$ & & $\checkmark$ & & & $\checkmark$ & & & & & & & & & $\checkmark$ & & & & & $\checkmark$ & \\
\hline $\begin{array}{l}\text { Khalilzadeh } \\
\text { et al. (2017) }\end{array}$ & UTAUT & $\begin{array}{l}\text { Behavioural } \\
\text { Intention }\end{array}$ & $\checkmark$ & & & $\checkmark$ & $\checkmark$ & $\checkmark$ & & & & & & & & & $\checkmark$ & & & $\checkmark$ & & & $\checkmark$ & $\checkmark$ & $\checkmark$ & $\checkmark$ & & \\
\hline Duane et al. (2014) & TAM & $\begin{array}{l}\text { Willingness } \\
\text { to make an } \\
\text { M-Payment }\end{array}$ & & $\checkmark$ & $\checkmark$ & $\checkmark$ & & & & & & & & $\checkmark$ & & & $\checkmark$ & & & & & & & & & & & \\
\hline $\begin{array}{l}\text { Al-Jabri and Sohail } \\
(2012)\end{array}$ & DOI & $\begin{array}{l}\text { Mobile Banking } \\
\text { Adoption }\end{array}$ & & & & & $\checkmark$ & & & $\checkmark$ & $\checkmark$ & $\checkmark$ & & & & & & $\checkmark$ & $\checkmark$ & & & & & & & & & \\
\hline Kim et al. (2010) & TAM & $\begin{array}{l}\text { Intention to Use } \\
\text { M-Payment }\end{array}$ & & $\checkmark$ & $\checkmark$ & & & & & $\checkmark$ & & & & $\checkmark$ & $\checkmark$ & & & & & & $\checkmark$ & $\checkmark$ & & & & & & \\
\hline $\begin{array}{l}\text { Koenig-Lewis } \\
\text { et al. (2015) }\end{array}$ & UTAUT2 & $\begin{array}{l}\text { Intention } \\
\text { to use }\end{array}$ & & $\checkmark$ & $\checkmark$ & & $\checkmark$ & & $\checkmark$ & & & & & & & & & & & $\checkmark$ & & & & & & & & \\
\hline Di Pietro et al. (2015) & $\begin{array}{l}\text { TAM, DOI, } \\
\text { UTAUT }\end{array}$ & $\begin{array}{l}\text { Intention to use, } \\
\text { Users' behaviour }\end{array}$ & $\checkmark$ & $\checkmark$ & $\checkmark$ & & & $\checkmark$ & & $\checkmark$ & & & & & & & & & & & & & & & & & & \\
\hline $\begin{array}{l}\text { Cheng and Huang } \\
\text { (2013) }\end{array}$ & TAM & $\begin{array}{l}\text { Behavioural } \\
\text { Intention }\end{array}$ & & $\checkmark$ & $\checkmark$ & & $\checkmark$ & & & & & & & $\checkmark$ & & & & & & & & & & & & & & \\
\hline
\end{tabular}

